\title{
Dual Effect of Nanomaterials on Germination and Seedling Growth: Stimulation vs. Phytotoxicity
}

\author{
Réka Szőllősi * (1) Árpád Molnár, Selahattin Kondak (1) and Zsuzsanna Kolbert \\ Department of Plant Biology, University of Szeged, H-6726 Szeged, Hungary; molnara@bio.u-szeged.hu (Á.M.); \\ selahattinkondak@gmail.com (S.K.); kolzsu@bio.u-szeged.hu (Z.K.) \\ * Correspondence: szoszo@bio.u-szeged.hu
}

Received: 4 November 2020; Accepted: 5 December 2020; Published: 10 December 2020

\begin{abstract}
Due to recent active research, a large amount of data has been accumulated regarding the effects of different nanomaterials (mainly metal oxide nanoparticles, carbon nanotubes, chitosan nanoparticles) on different plant species. Most studies have focused on seed germination and early seedling development, presumably due to the simplicity of these experimental systems. Depending mostly on size and concentration, nanomaterials can exert both positive and negative effects on germination and seedling development during normal and stress conditions, thus some research has evaluated the phytotoxic effects of nanomaterials and the physiological and molecular processes behind them, while other works have highlighted the favorable seed priming effects. This review aims to systematize and discuss research data regarding the effect of nanomaterials on germination and seedling growth in order to provide state-of-the-art knowledge about this fast developing research area.
\end{abstract}

Keywords: nanomaterials; seed germination; root elongation; seed priming; phytotoxicity

\section{Introduction}

The term "nanomaterial" (NM) refers to a material with one dimension under $100 \mathrm{~nm}$ [1-4]. With the development of nanotechnology, the use of nanomaterials is seeing an unprecedented increase, and studies are needed to focus on the effects of nanomaterials in all living organisms, especially sessile plants that cannot avoid these kinds of external factors. It is also essential to evaluate the possible hazards of nanomaterials in the environment, as well as in plants, animals and humans, because of their increasing emissions. For example, the global output of $\mathrm{ZnO}$ nanoparticles (NPs), which are widely applied, is between 550 and 5550 tons per year, a value that is approximately 10-100 times higher than that of other NMs [5].

Most of the physico-chemical properties of NMs vary depending on shape, size, surface area, surface/volume ratio, chemical behavior, particle charge, production method, coating, and so on, as has previously been described in detail [6,7]. Changes in NM synthesis can lead to magnetic properties in NMs that can be useful in medical processes [8]. NMs are often modified with oxides or other molecules to increase conductivity and help avoid aggregations of NMs, and this has a significant impact on NM behavior [9]. According to their dimensionality, NMs can be divided into four categories: OD NMs, where the electrons are confined in all three dimensions (e.g., quantum dots (QDs)); 1D NMs, where the electrons can move in one dimension (e.g., quantum wires and nanofibers); 2D NMs, where the electrons can move in two dimensions (mostly nanofilms and nanosheets); and 3D NMs, which are usually made of other NMs and allow electrons to move freely in all three dimensions [10]. The morphology of NMs is diverse, from nanocubes and nanopyramids to nanowires and nanozigzags. NMs can be composed of a single material, or they can be used as composites. The uniformity of NMs, especially nanoparticles, can be isometric if all particles are roughly the same size or inhomogeneous. This attribute will 
influence the behavior of agglomerated NPs in solution [9]. Based on these features and previously published reports, we suppose that each NM may have specific impacts (either beneficial or toxic) on living organisms like plants; therefore, NMs must be characterized in every case study.

Classification of NMs is depicted in Figure 1. The difference between properties of NM groups is significant, and all main NM groups (i.e., carbon-based, metal-based NMs, quantum dots, dendrimers and nanocomposites) will be described later in detail.

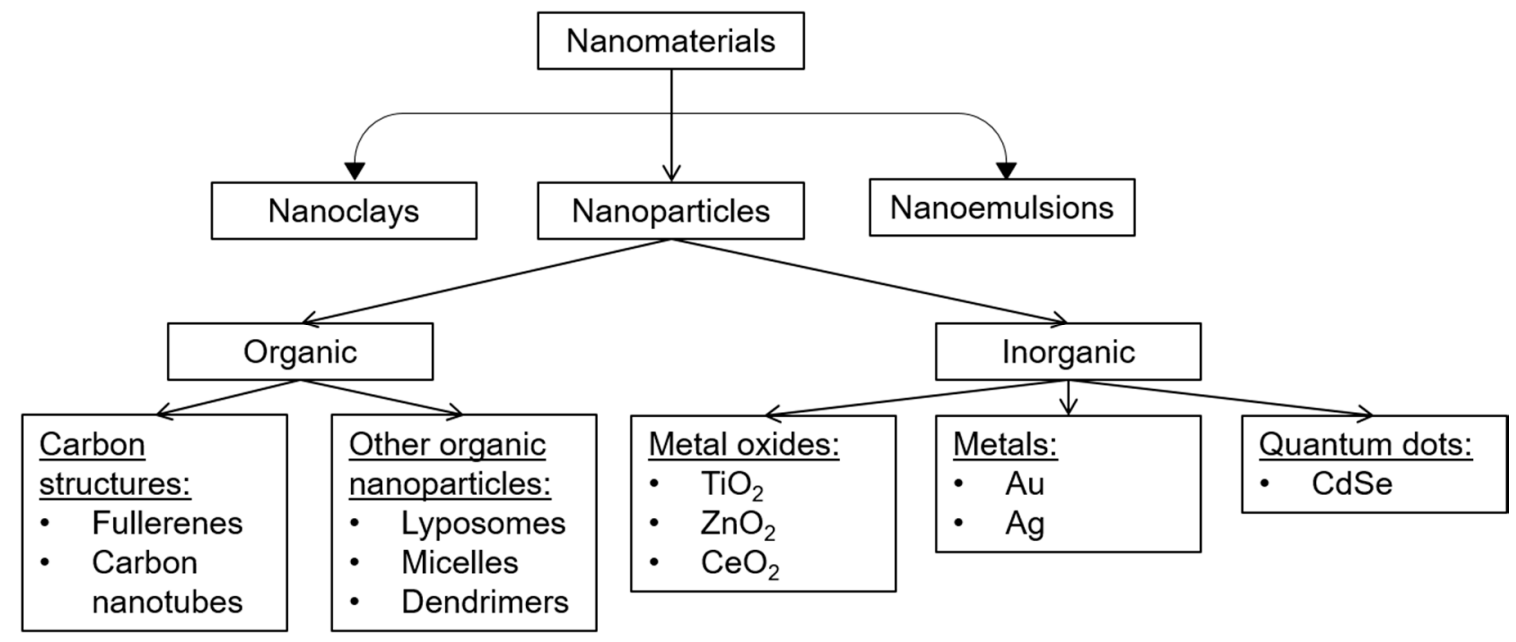

Figure 1. Classification of different nanomaterial (NM) groups (according to [7], with modifications).

Nanofibers (NFs) are a relatively new member of one-dimensional nanomaterial, like nanotubes or nanowires. Their large surface areas, high tensile strength and porosity make them interesting to the industry. NFs are made cost effectively through electrospinning, a method devised in the USA in 1902 [11]. With recent advances in electrospinning, both synthetic and natural polymers are usable in the production of NFs. It is worth mentioning that, besides polymeric NFs, ceramic and metal oxide NFs can also be created. The morphology, diameter (from 10 to several hundred nanometers), chemical composition and surface modifications of NFs can be modified [12]. New advances have made nanofibers a promising material for medical and cosmetic applications such as tissue or organ repair [13].

Nanoclays and organoclays are a type of silicate with general thickness of 50-200 nm. They are produced utilizing the hydrophilic nature of clay molecules via an ion exchange reaction in the aqueous or solid state. During the reaction, the gap between clay layers is widened, enabling organic cation molecule integration between the layers. The surface of the clay sheets also changes from hydrophilic to hydrophobe. These NMs have antimicrobial and toxin absorption capabilities, making them ideal for applications in food industry (food packaging) [14].

Emulsions are defined as the dispersion of two immiscible liquids [15]. Nanoemulsions are made in a viscous liquid via the dispersion of polymers, droplets or other solid materials, and is referred to as dispersed or discontinuous phase. Physical properties of these liquids, such as viscosity, phase behavior and density are influenced by oil phase components [16]. These stable colloids are usually used in the food industry to develop biodegradable food packaging, to increase the shelf lives of foods and as a decontaminant for equipment [17].

The word "nanoparticle" (NP) was coined recently and usually refers to manufactured anthropogenic NPs. By definition, NPs have at least two dimensions between 1-100 nm, which include naturally occurring particles (e.g., dust, aerial particles, colloids, etc.). In nature, these structures are common and have been identified in glacial ice cores and the Cretaceous-Tertiary boundary layer [18-20]. In samples of this layer from Italy, magnetic iron materials, hematite and silicate has been found with sizes between 16-27 nm [19]. The origins of different NPs are summarized in Figure 2. 


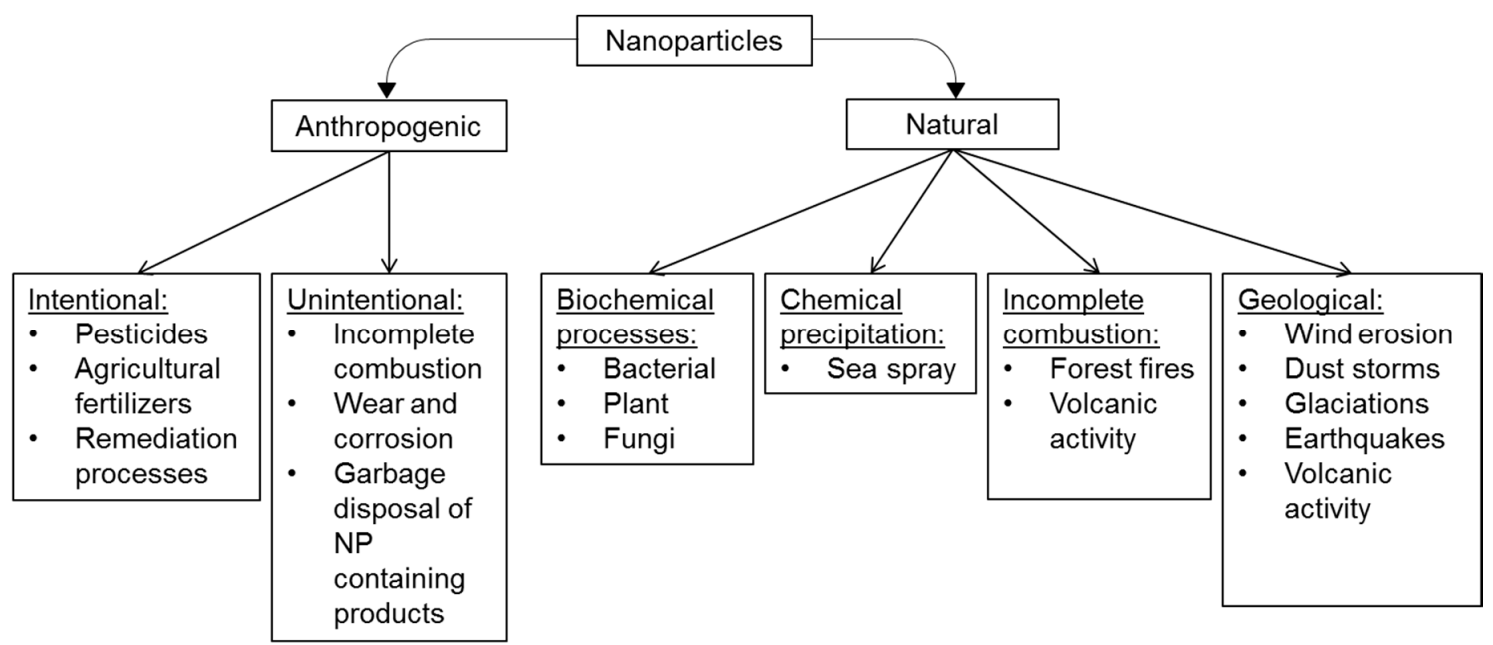

Figure 2. The origin of different nanoparticles according to Buzea és Pacheco [21] with modifications. The abbreviation NP refers to 'nanoparticle'.

In this study, due to their relatively fresh appearance and widespread use compared to natural NPs, anthropogenic NPs are discussed. Manufactured NPs include organic forms, such as carbon structures, polymers, dendrimers, lyposomes and micelles, while inorganic forms include metal oxides, metals and quantum dots (QDs). Compared to their bulk forms, their physical and chemical characteristics differ greatly [22], as their unique nanostructures have excellent properties.

NPs are widely used across the industries and the rapid market increase in the previous decade was predicted $[23,24]$. The growth of their production and disposal has enhanced the possibility of NPs being released into the environment and coming into contact with living organisms, such as plants $[22,25,26]$. Studies dealing with the effects of NMs establish that many factors can have an impact on the exact outcome of the NM-plant interactions, including the plant species, the size of the applied NMs, the duration or existence of pre-cultivation (e.g., seed priming), the concentration and span of NM exposure or the growing conditions, namely the germination test performed in Petri dishes or hydroponics or a pot experiment using soil. To date, it has been well documented by reviews and case studies that how several NMs, mainly metallic NPs, may positively or negatively influence biomass, the photosynthetic activity or the yield of adult plants, but there remains a great lack of knowledge concerning the early developmental stage, i.e., seed germination and early seedling growth [27-29]. The uptake of NMs by mature (adult) plants has been documented by several studies. A large number of studies reported ZnO NPs entering plant tissues or cells [22,30-32]. In case of carbon-based NPs, single-walled carbon nanotubes (SWCNTs) have been identified inside plant tissues [33,34]. A recent report has reviewed in detail how carbon-based NMs can be uptaken by root or shoot (due to foliar application), translocated via the vascular tissues and affect plant growth or modulate stress tolerance (summarized by [35]). However, most NMs and especially NPs have been reported in studies as materials entering plant tissues (overviewed by [36]), thus further research has to be conducted to reach a conclusion on this topic, due to the diversity of NMs in terms of their size and morphology.

Since seed germination is the first step and the most sensitive stage of higher plants' ontogenesis, studying the effects of NMs during this phase seems to be very informative for researchers and agronomists. Since 2000, a growing number of reports ( $>1000$, Science Direct, [37]) have analyzed the impact of metal-containing nanosized materials on seed germination, especially the positive/negative effects of NPs which are broadly applied in agriculture, electronics, cosmetics, medicine, etc. (overviewed by [38-40]).

During seed germination, the NMs (NPs or QDs) first have to penetrate the seed coat which generally contains sclerenchyma, namely sclereids, and due to its physical-chemical integrity, it can act as a barrier for the NMs [41]. Some reports suggest that NMs use the intercellular spaces of the tissues 
or create new pores mainly through the upregulation of aquaporin production (discussed by $[38,42]$ ). Another recent study has also confirmed that some metal oxide NPs $\left(\mathrm{ZnO}\right.$ and $\left.\mathrm{TiO}_{2}\right)$ can get through the seed coat and provoke the embryonic differentiation via stimulating the enzymes which are involved in interrupting seed dormancy [38]. When the radicle of the new progeny emerges, the developing tissues of the root apex get in touch with the NMs which may enter the rhizodermis via apoplastic transport, endocytosis or other carriers, then within the root they flow toward the vascular cylinder using symplastic pathways and are translocated to other progressing plant parts (discussed by [28,43]).

Naturally, it is also important to know whether the uptaken NMs are biotransformed into their ionic form, and whether they can be detected in plant tissues in nano-form, as it was reviewed by $[27,28,43]$.

Up to now, in most experiments, germination tests have been executed in Petri dishes applying NM-containing agar or wet filter paper, and it has been assessed that the exposition to NMs may affect the efficiency of germination (with parameters like germination percentage, mean germination time or seedling vigor index) and the early plant growth (radicle/root length and plumule/shoot length, see [44]), as we will discuss it in this review.

\section{Effects of Nanomaterials on Seed Germination and Seedling Growth}

\subsection{Concentration-Dependent Effects of CNMs on Seed Germination and Seedling Growth}

The first carbon-based nanomaterial was a 60-carbon atom fullerene, discovered in 1985 [45]. In 1991, a fullerene product, carbon nanotubes (CNTs), were first manufactured [46]. The synthesis of carbon nanotubes continued with multi-walled carbon nanotubes (MWCNTs) with $10 \mu \mathrm{m}$ length and 5-40 $\mathrm{nm}$ diameter. With added cobalt-nickel catalyst, the production of single-walled carbon nanotubes (SWCNTs) was achieved. The strength to weight ratio of SWCNTs is 460 times larger compared to steel. Nowadays, the types of carbon NMs are numerous: fullerenes and fullerene cages, SWCNTs and MWCNTs, cup-stacked carbon nanotubes, graphene sheets, etc. [46-49]. Most carbon NPs are hydrophobic, leading to an aggregation or precipitation in aqueous solutions. Due to the large differences in morphology and chemical properties, individual carbon-based NPs are a diversified group, with large industrial usage among NMs. Due to their large usage, there is a growing concern that CNTs behave similarly to asbestos and are harmful to human health [50].

\subsubsection{Carbon Nanotubes (CNTs)}

In the early work of Lin and Xing [51], the influence of MWCNTs on seed germination and seedling growth of six different crop species such as radish (Raphanus sativus), rapeseed (Brassica napus), ryegrass (Secale cereale), lettuce (Lactuca sativa), maize (Zea mays) and cucumber (Cucumis sativus) was evaluated. Germination was not affected by MWCNTs in either of the examined species but seedling root growth was enhanced in ryegrass and maize [51]. Similarly, MWCNTs (1000 $\left.\mathrm{mg} \mathrm{L}^{-1}\right)$ had no effect on the germination process of zucchini (Cucurbita pepo) and carrot (Daucus carota) [51,52]. However, MWCNTs at the concentration range of $10-40 \mathrm{mg} \mathrm{L}^{-1}$ notably enhanced seed germination and seedling growth in tomato (Solanum lycopersicum) [53] and the promoting effect of MWCNTs was supposed to be due to their capability of penetrating the seed coat and promoting water uptake. MWCNTs stimulated cell growth in tobacco BY2 cell suspension, which was accompanied by the upregulation of genes involved in cell division (CycB)/cell wall formation (NtLRX1, extensin) and water transport (NtPIP1, aquaporin), providing a molecular explanation for the growth-inducing effect of MWCNTs [54]. The work of Cañas et al. [55] compared the effects of nonfunctionalized and functionalized (with poly-3-aminobenzenesulfonic acid) SWCNTs and observed that the effect was dependent on the plant species, since the root growth was not affected in cabbage (Brassica oleracea) and carrot, but was inhibited in tomato, while it was promoted in onion (Allium cepa) and cucumber seedlings. It was also observed that nonfunctionalized CNTs affect root length more seriously than in their functionalized form. Using tobacco BY2 cells, Liu et al. [56] convincingly showed that SWCNTs are able to penetrate through the plant cell wall and plasma membrane, supporting the observed effects on 
seedling growth, which is probably due to their size (1-15 nm), often smaller compared to MWCNTs [57]. Several further reports have indicated that seed germination and/or seedling growth is induced by MWCNTs and SWCNTs in plant species like the tomato, Indian mustard (Brassica juncea), onion, radish (Raphanus sativus), turnip (Brassica rapa), sage (Salvia officinalis), pepper (Capsicum annuum), tall fescue (Festuca arundinacea), wheat (Triticum aestivum), maize, peanut (Arachis hypogaea), garlic (Allium sativum), rice (Oryza sativa), barley (Hordeum vulgare) soybean (Glycine max), switchgrass (Panicum virgatum), and gram (Cicer arietinum) [58-66], as reviewed by [67] and [68]. The concentration-dependent effect of MWCNTs, Fe-filled carbon nanotubes (Fe-CNTs), and Fe-Co-filled carbon nanotubes (FeCo-CNTs) was compared in the study of Hao et al. [69], where the seedling's root length was increased by the low concentrations. However, auxin (IAA) content in rice roots and shoots decreased upon the exposure to all of the three CNTs at all concentrations. Additionally, CNT treatment resulted in decreased levels of other phytohormones including gibberellin (GA1+3), cytokinin (IPA), jasmonic acid (JA), brassinolide (BR), and abscisic acid (ABA). These changes in hormonal status may contribute to the negative effects of the examined CNTs [69]. Moreover, in case of Hyosciamus niger, MWCNTs decreased the germination percentage and increased the germination time and the early seedling growth was decreased as well [70]. MWCNT treatment caused oxidative stress, which was supported by the elevation of lipid peroxidation, electrolyte leakage, $\mathrm{H}_{2} \mathrm{O}_{2}$ and also by the activation of the antioxidant defense [70]. These results were supplemented by Khalifa [71], who observed that the toxic effects of high MWCNT

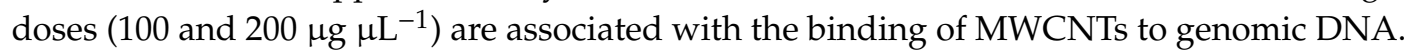

In contrast, the application of CNTs increased the germination rate of $P$. virgatum seeds and speeded up the germination process of sorghum (Sorghum bicolor) seeds as well as promoted seedling growth [72]. A similar positive effect of CNTs on tomato seedling growth was observed by [73] where modified antioxidant response and the increased production of antioxidant compounds were found. Seed priming with MWCNTs functionalized with carboxylic acid (MWCNT-COOH) proved to be effective in improving seed germination and seedling vigor in buffaloberry (Shepherdia canadensis) and green alder (Alnus viridis) [74]. An important novel finding of this study was that the cessation of both embryo and seed coat dormancy was associated with the remodeling of C18:3-enriched fatty acids in seed membrane lipid molecular species, suggesting that MWCNTs functionalized with carboxylic acids modulates cell membrane lipid metabolism [74]. The concentration-dependent effect of CNTs (and graphene) was further supported in tomato seedlings where seed priming had no effect on the germination process, but increased root biomass and activated antioxidants (ascorbic acid, phenols, flavonoids, superoxide dismutase (SOD), catalase (CAT), GPX (glutathione peroxidase), etc.) [75].

Recently, the stress modulating effect of CNTs was investigated by several research groups. It was reported that MWCNT treatment aggravated the negative effects of cadmium (Cd) on root elongation, lateral root and root hair formation, root and shoot biomass formation and $\mathrm{Cd}$ accumulation was induced by MWCNTs [76]. In case of drought-stressed Glycine max seeds, however, SWCNTs improved germination and seedling growth by reducing lipid peroxidation and $\mathrm{H}_{2} \mathrm{O}_{2}$ content but increasing ascorbic acid (AsA) content and SOD, CAT, peroxidase (POD) activities suggesting that SWCNTs may play an important role in the improvement of antioxidant capacity of soybean seedlings under drought stress [77]. In the work of Baz et al. [78], twenty-seven varieties of L. sativa (lettuce) were compared for their sensitivity to salt stress, and the seeds were pre-treated with CNTs. Pre-treatment with CNPs significantly improved seed germination in the case of salt exposure $(150 \mathrm{mM} \mathrm{NaCl})$, and high temperature; however, different lettuce varieties exhibited distinct responses to nanoparticle treatments drawing attention to the genotype-dependent effect of CNTs [78].

The large amount of experimental data indicates that the effect of MWCNTs and SWCNTs on seed germination and seedling growth shows concentration dependence, dependence on the plant species, on the plant genotype and also on the treatment conditions. Therefore, the optimal circumstances and growth-promoting concentrations are recommended to be experimentally verified before practical application. 


\subsubsection{Carbon Nanodots (CDs)}

As for the effect of carbon dots (CDs) on seed germination and seedling growth, there are only few results available. The first study was conducted on Zea mays plants where high doses of CDs (1000 and $2000 \mathrm{mg} \mathrm{L}^{-1}$ ) led to decreased root and shoot biomass due to $\mathrm{H}_{2} \mathrm{O}_{2}$ accumulation and intensified lipid peroxidation. Additionally, CD exposure activated antioxidant enzymes like CAT, APX, GPX and SOD. CDs were visualized in root-cap cells, cortex cells and vascular bundle of roots and also in leaf mesophyll, indicating the effective absorption and translocation of CDs in maize. Interestingly, the excretion of CDs from leaf blade was also observed [79]. Using a wide concentration range of CDs (0.02-0.12 $\mathrm{mg} \mathrm{mL}^{-1}$ ) for treating mung bean (Vigna radiata) sprouts, a concentration-dependent effect was observed since the sprouts showed root and stem elongation, increased biomass production and carbohydrate content as the effect of low CD doses. Additionally, CDs enhanced RuBisCO activity and chlorophyll content in the sprouts, suggesting improved photosynthesis [80]. In another study, V. radiata sprouts were cultivated in the presence of N-doped C-dots (N-CDs) and a significant enhancement in the sprouts' yield was observed compared to the aqueous control [81], indicating the effectiveness of N-CDs as a nitrogen nanofertilizer. Qian et al. [82] compared the in planta distribution and the effects of three types of CDs (bared CDs, CD-PEI (modified by polyethylenimine), and CD-PAA (modified by polyacrylic acid)] on growth of $C$. pepo seedlings. It was found that all three types of CDs triggered the antioxidant defense systems (SOD, POD, CAT) [82]. The available literature has recently been reviewed by [83]. Furthermore, in a comparative study, the most significant promoting effect of functional CDs (FCNs), possessing the largest number of functional groups and a small size, on the growth of Arabidopsis thaliana seedlings, was observed. The remarkable effect of FCNs may be due to their perfect aqueous dispersity, nutrient adsorption capacity and bioaffinity, as suggested by the authors [84].

\subsubsection{Carbon Nanohorns (CNHs)}

Carbon nanohorns (CNHs) are a promising carbon-based nanosized material with special characteristics. Unlike carbon nanotubes, $\mathrm{CNHs}$ are uniform in size and can be well dispersed in solvents. Moreover, they can be synthesized in large quantities without any catalyst [65]. The germination and growth-promoting effect of single-walled carbon nanohorns (SWCNHs) were evidenced at the physiological, cellular and genetic levels in the study of Lahiani et al. [64] using barley, maize, soybean, rice, switchgrass and tomato seeds. The germination of barley and soybean showed only a slight response to SWCNHs, while the germination percentage of corn, rice, tomato and switchgrass significantly improved under the effects of all three SWCNHs concentrations $\left(25,50\right.$ or $\left.100 \mathrm{mg} \mathrm{L}^{-1}\right)$ compared to the control [64]. As for the seedling development, SWCNHs exerted inducing effects on shoot and root length, leaf number, as well as fresh and dry weights, however, the effects proved to be concentration-dependent and were dependent on the plant species. This study also confirmed that the growth of tobacco cells is induced by SWCNHs and that SWCNHs are able to affect the expression of a number of tomato genes that are involved in stress responses, cellular responses and metabolic processes [64]. Recently, the effect of SWCNHs on the root system growth of Arabidopsis thaliana seedlings was evaluated at the molecular and metabolic levels [85]. A low concentration of SWCNHs $\left(0.1 \mathrm{mg} \mathrm{L}^{-1}\right)$ promoted primary root (PR) elongation and lateral root (LR) formation, as well as increased the lengths of the meristematic and elongation zones. It was further confirmed that SWCNHs enhanced stem cell niche activity, meristematic cell division potential and the auxin level and signaling of Arabidopsis root apex. Metabolomics supported by transcriptomic data revealed that SWCNHs reprogrammed carbon/nitrogen metabolism and increased the levels of secondary metabolites (e.g., serotonin, hypoxanthine, adenine). These data provide insight into the molecular basis of the growth promoting effect of SWCNHs [85]. 


\subsubsection{Fullerenes and Fullerols}

In the work of Liu et al. [86], the water-soluble fullerene malonic acid derivative (FMAD) inhibited the root and hypocotyl elongation of Arabidopsis seedlings in a concentration-dependent manner, although the germination capacity was not affected, possibly due to the protective effect of the seed coat. The observed root-shortening effect of FMAD is associated with the disruption of cell division, microtubule arrangement, auxin levels and with intracellular ROS (reactive oxygen species) accumulation. In contrast, polyhydroxy fullerene (PHF or fullerol) treatment at high concentrations $\left(100,000\right.$ and $\left.200,000 \mathrm{mg} \mathrm{L}^{-1}\right)$ exerted a significant positive effect on the root and hypocotyl elongation of Arabidopsis seedlings [87]. In addition, PHF promotes the elongation of barley roots due to the enhancement of their longitudinal extensibility in the elongation root zone [88]. Additionally, in the presence of a stressor such as UV-B radiation, salt stress or the presence of a high salicylic acid dosage, PHF exerted a more pronounced effect on root growth. PHF protected seedlings from oxidative damage induced by UV-B irradiation, suggesting that PHF is able to enhance growth due to its ROS scavenging capacity [88]. Xiong et al. [89] applied seed priming with PHF and observed a significant inducing effect on seed germination in the case of polyethylene glycol (PEG)-triggered osmotic stress. Additionally, the foliar application of PHF led to an increment in shoot dry weight and photosynthesis in rapeseed (Brassica napus) seedlings grown in dried soil. The level of ROS decreased and the content of antioxidants as well as the activities of antioxidant enzymes increased in PHF+drought-treated seedlings compared to seedlings exposed to drought alone. It was also observed that the PHF treatment of drought-stressed seedlings induced an elevated $A B A$ content in the leaves and triggered $A B A$ biosynthesis by downregulating the expression of the ABA catabolic gene CYP707A3. In a recent study, the protein profile of maize seeds during fullerene-influenced germination was examined [90]. Maize seeds showed to have a higher germination rate and faster germination due to the effect of the water-soluble quaternary ammonium salts of iminofullerenes (IFQA). Upon IFQA treatment storage, proteins (e.g., globulin, vicilin-like embryo storage protein) were downregulated and proteins involved in energy production (e.g., glyceraldehyde-3-phosphate dehydrogenase 2) and sugar metabolism (e.g., UDP-glucose 6-dehydrogenase isoform 2) were upregulated, explaining a faster germination.

\subsubsection{Graphene and Graphene Oxide (GO)}

Due to its special characteristics, graphene has great potential in industrial, biomedical and agricultural applications. Therefore, the effects of graphene and graphene oxide $(\mathrm{GO})$ on the germination and seedling growth were evaluated by several authors. For instance, Nair et al. [91] revealed that Oryza sativa seedlings, germinated in the presence of graphene, showed better viability and growth compared to untreated seedlings. Similarly, the germination capacity of tomato seeds was increased by powdered graphene possibly due to the ability of graphene to improve water uptake via the seed coat [92]. On the other hand, several studies reported that seed germination was delayed and/or inhibited by graphene or GO application. For instance, $O$. sativa seed germination was delayed by increasing graphene concentrations (5-200 $\mathrm{mg} \mathrm{L}^{-1}$, [93]). In another short-term study, graphene $\left(250,500,1000\right.$ and $\left.1500 \mathrm{mg} \mathrm{L}^{-1}\right)$ significantly improved root elongation, but inhibited root hair development, which may be associated with graphene induced-oxidative stress in the roots of wheat seedlings [94]. In maize seedlings, sulfonated graphene NPs at low concentration (50 $\mathrm{mg} \mathrm{L}^{-1}$ ) stimulated growth (plant height, root and shoot biomass), while a high dosage (500 $\mathrm{mg} \mathrm{L}^{-1}$ ) exerted a strong inhibitory effect accompanied by $\mathrm{Ca}^{2+}$ signaling, ROS production and lipid peroxidation [95]. During the comparison of the effects of $\mathrm{GO}$ and amine-modified graphene $\left(\mathrm{G}-\mathrm{NH}_{2}\right)$ it was found that at high concentrations (500, 100 or $2000 \mathrm{mg} \mathrm{L}^{-1}$ ), GO inhibited wheat germination and seedling growth, while the same doses of $\mathrm{G}-\mathrm{NH}_{2}$ exerted positive effects. The electrolite leakage of roots was increased by GO exposure supporting the toxic nature of this nanomaterial type [96]. According to Vochita et al. [97], wheat seed germination was inhibited by a high dosage of GO (2000 mg L $\left.{ }^{-1}\right)$ and a slight reduction in root elongation was also observable at this concentration. Moreover, the increment in chromosomal aberrations and mitotic abnormalities indicates the clastogenic and aneugenic effect of 
GO in wheat root meristem. Recently, Xu et al. [98] have claimed that $10 \mathrm{~nm}$-sized graphene quantum dots (GQDs) can promote the absorption of water and nutrients by increasing the effective surface areas of the root epidermal (rhizodermal) cells. Their schematic model shows that GQDs directly attach to the surfaces of the plant root cells, growing absorptive area for the ions on the root surface, but there is no information about the mode of penetration and further transport within the root.

The presented examples clearly show that the effect on early plant development depends on the concentration of graphene or GO. Due to its capability in transporting water, graphene improves seed germination; however, elevated doses cause oxidative stress and genotoxicity.

The promoting influences of carbon-based NMs (CNMs) on seed germinations are summarized in Figure 3.

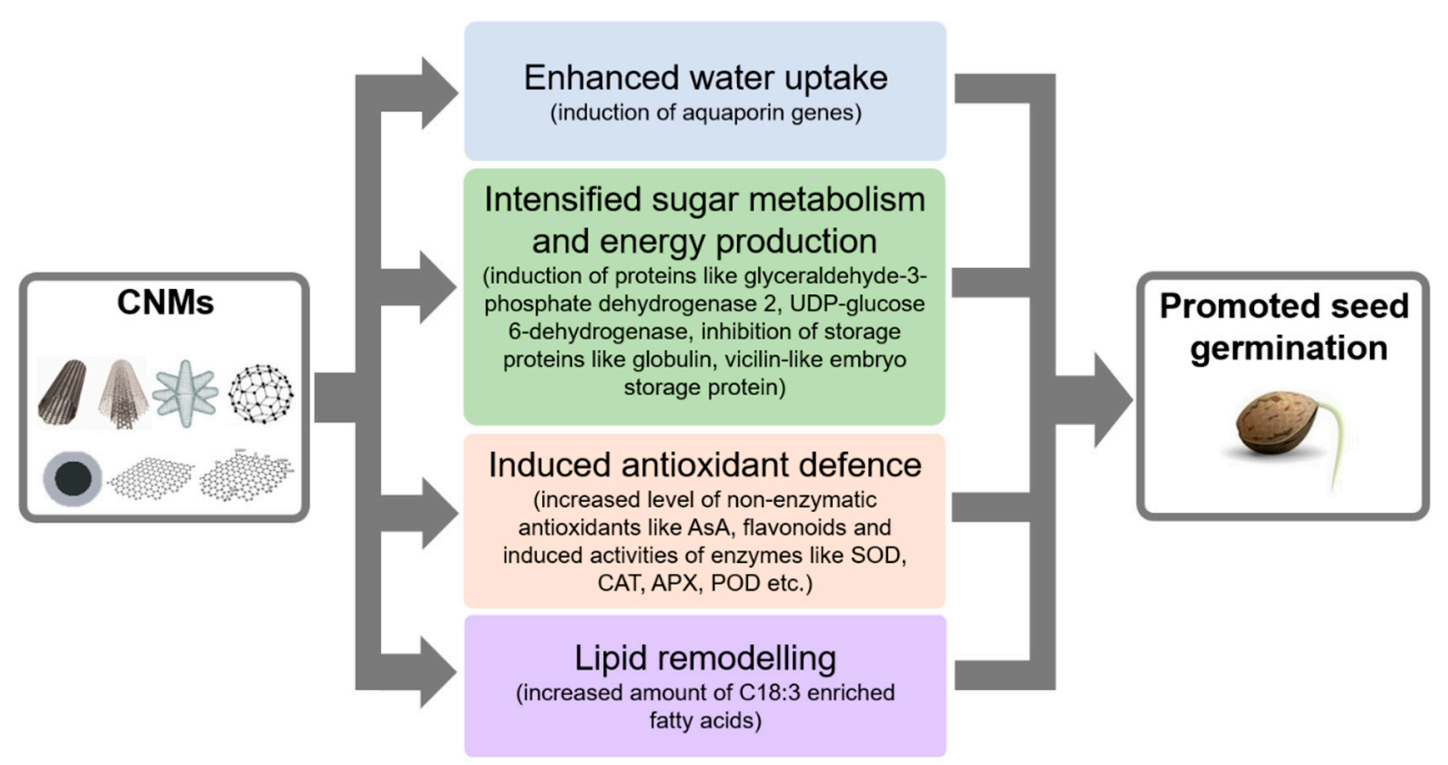

Figure 3. Biochemical and molecular mechanisms of the germination-promoting effect of carbon nanomaterials (CNMs) described so far. Upon CNM exposure, enhanced water uptake, intensified sugar metabolism/energy production, induced antioxidant defense and the remodeling of membrane lipids in seeds have been described in different experimental systems. See details in the text. Abbreviations: AsA, ascorbic acid; SOD, superoxide dismutase; CAT, catalase; APX, ascorbate peroxidase; POD, peroxidases.

\subsection{The Influences of Metal-Based NMs on Germination and Seedling Growth}

The effect of metallic nanomaterials (NMs) (including metal and metal oxide NPs, and quantum dots, QDs) on plant development and physiological processes is an intensely researched area, since plants being the first step of food web have a key role in a potential NM contamination. It was demonstrated that, e.g., QDs, known as nanoscale autofluorescent semiconductors, are not only uptaken by plants like Arabidopsis but are transferred to its herbivores, as well [99]. Moreover, seed germination, including the emergence of the radicle and the elongation of the primary root, is the most sensitive part of the plant life cycle, therefore both the beneficial and negative effects of metallic NMs can be well tested.

The use of metals and metal oxides dates back to ancient times, as titanium oxide $\left(\mathrm{TiO}_{2}\right)$ was used as paint in ancient Egypt. $\mathrm{TiO}_{2}$ and zinc oxide $(\mathrm{ZnO}) \mathrm{NPs}$ are widely used across the industry. Their high surface area compared to their weight and volume, high reactivity and high chemical mechanical and heat stability resulted in a diverse use for all metal oxide NPs, especially ZnO. Metal oxide NPs are used in sunscreens, paint and solar cells, laser technology, etc. Interestingly, the yearly production of ZnO NPs is estimated to be 10-100 times larger than other NMs [5]. Zero valent metal NPs are made with the reduction of metal salts, where the reductant type and conditions affect the physical properties of the NPs [100]. Zero valent iron has been used as a detoxifier against nitrates in remediation processes 
and new research suggests organochloride pesticides as a new remediation target [101]. Silver NPs are widespread in the industry [102] and used in air filters, washing machines, baby products and wound dressing. Both the metallic silver NPs and ionic silver have been used extensively. Nanosized silver is reactive in aqueous solutions, resulting in the short half-life of the active form. This resulted in the absorption of silver NPs on other macroparticles, resulting in a stable colloidal form which is still referred to as nano silver by the manufacturers $[103,104]$. In medical applications, gold nanocolloids are not rare and nanoparticulate gold is used in electronics and as a catalyst.

\subsubsection{Metallic NPs}

Similarly to other NMs, metal-containing NPs have been shown to have dual effects in plants, including seed germination. Beneficial influences of elemental metallic NP application were displayed in some crops. The germination of cucumber and lettuce seeds was promoted by solutions containing $62 \mu \mathrm{g} \mathrm{mL}^{-1} \mathrm{Au}$ NPs for 7 days [105], and similar results were found in the case of Pennisetum glaucum (pearl millet) after soaking the seeds for $2 \mathrm{~h}$ in Au NP (20 and $\left.50 \mu \mathrm{g} \mathrm{mL}^{-1}\right)$ ([106]; Table 1). 
Table 1. Positive effects of metal and metal oxide NPs on seed germination and seedling growth.

\begin{tabular}{|c|c|c|c|c|c|c|c|c|c|}
\hline & Plant Name & Size of NM & $\begin{array}{c}\text { Type and } \\
\text { Chemical } \\
\text { Composition } \\
\text { of NM }{ }^{*}\end{array}$ & $\begin{array}{l}\text { Duration of } \\
\text { Pre-Cultivation }\end{array}$ & $\begin{array}{l}\text { Conc. of the NM } \\
\text { Exposure }\end{array}$ & $\begin{array}{c}\text { Time of } \\
\text { Exposure }\end{array}$ & $\begin{array}{l}\text { Growth } \\
\text { Conditions }\end{array}$ & $\begin{array}{l}\text { Main Effects on Germination and } \\
\text { Early Growth ** }\end{array}$ & Reference \\
\hline \multirow{13}{*}{ 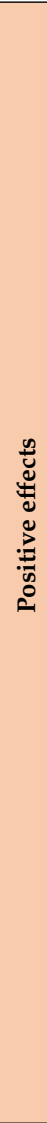 } & Cucumis sativus $\mathrm{L}$. & \multirow{2}{*}{$10.1 \pm 4.2 \mathrm{~nm}$} & \multirow{2}{*}{$\mathrm{Au} \mathrm{NP}$} & \multirow{2}{*}{ - } & \multirow{2}{*}{$62 \mu \mathrm{g} \mathrm{mL} L^{-1}$} & \multirow{2}{*}{7 days } & \multirow{2}{*}{$\begin{array}{c}\text { Petri dishes } \\
\text { (germination } \\
\text { test) }\end{array}$} & Germination index $\uparrow \mathrm{ns}$ & \multirow[t]{2}{*}{ [105] } \\
\hline & Lactuca sativa L. & & & & & & & Germination index $\uparrow$ & \\
\hline & Pennisetum glaucum $\mathrm{L}$. & $14-35 \mathrm{~nm}$ & $\mathrm{Au} \mathrm{NP}$ & $\begin{array}{l}\text { Seed soaking } \\
\text { for } 2 \mathrm{~h} \text { in test } \\
\text { solution }\end{array}$ & 20 and $50 \mu \mathrm{g} \mathrm{mL} L^{-1}$ & 5 days & $\begin{array}{c}\text { Petri dishes } \\
\text { (germination } \\
\text { test) }\end{array}$ & $\begin{array}{c}\text { Germination } \% \uparrow \mathrm{ns} \text { and } \uparrow \text {; total } \\
\text { seedling length } \uparrow \mathrm{ns}\end{array}$ & [106] \\
\hline & Cucurbita pepo L. & \multirow{2}{*}{$20 \mathrm{~nm}$} & \multirow{2}{*}{$\mathrm{Ag} \mathrm{NP}$} & \multirow{2}{*}{$\begin{array}{l}2 \mathrm{~h} \text { seed } \\
\text { priming }\end{array}$} & \multirow{2}{*}{$0.05-2.5 \mathrm{mg} \mathrm{L}^{-1}$} & \multirow{2}{*}{$\begin{array}{l}12-16 \text { days } \\
\text { after priming }\end{array}$} & $\begin{array}{l}\text { Petri dishes } \\
\text { (germination } \\
\text { test) }\end{array}$ & $\begin{array}{l}\text { Germination } \% \uparrow \text { at } 0.5-2.0 \mathrm{mg} \mathrm{L}^{-1} \\
\text { conc.; root length } \uparrow \text { at } 0.05-1.5 \mathrm{mg} \mathrm{L}^{-1}\end{array}$ & \multirow[t]{2}{*}{ [107] } \\
\hline & Citrullus lanatus L. & & & & & & & $\begin{array}{l}\text { Germination } \% \uparrow \text { at } 0.5-2.0 \mathrm{mg} \mathrm{L}^{-1} \\
\text { conc.; root length } \uparrow \text { at } 1-2.5 \mathrm{mg} \mathrm{L}^{-1}\end{array}$ & \\
\hline & Lolium multiflorum $\mathrm{L}$. & $\begin{array}{c}\text { Width: } \\
122 \pm 35 \mathrm{~nm}, \\
\text { length: } \\
11,908 \pm 6703 \mathrm{~nm}\end{array}$ & $\mathrm{Ag} \mathrm{NW}$ & - & $10 \mathrm{ppm}$ & 6 days & $\begin{array}{l}\text { Petri dishes } \\
\text { (germination } \\
\text { test) }\end{array}$ & $\begin{array}{c}\text { Root length } \uparrow \mathrm{ns} \text { and physical } \\
\text { separation from NWs caused further } \\
\text { increment }\end{array}$ & [108] \\
\hline & $\begin{array}{l}\text { Phaseolus vulgaris L. } \\
\text { 'Bali' and 'Delfina' }\end{array}$ & $\sim 10 \mathrm{~nm}$ & Ag NP & $\begin{array}{l}\text { Seed priming } \\
\text { for } 1.5 \mathrm{~h}\end{array}$ & $\begin{array}{l}0.25,1.25 \text { and } \\
2.5 \mathrm{mg} \mathrm{L}^{-1}\end{array}$ & 5 days & $\begin{array}{l}\text { Petri dishes } \\
\text { (germination } \\
\text { test) }\end{array}$ & Germination $\% \uparrow$ at all conc. & [109] \\
\hline & Zea mays L. & $<50 \mathrm{~nm}$ & $\mathrm{Co}_{3} \mathrm{O}_{4}$ & - & $\begin{array}{l}269.3-1000 \mathrm{mg} \mathrm{kg}^{-1} \\
\text { soil (DW) }\end{array}$ & 14 days & $\begin{array}{c}\text { Germination } \\
\text { test in pot } \\
\text { experiment }\end{array}$ & Germination $\% \uparrow n s$ at all conc. & [110] \\
\hline & Alyssum homolocarpum & \multirow{3}{*}{$10-25 \mathrm{~nm}$} & \multirow{3}{*}{$\mathrm{TiO}_{2} \mathrm{NP}$} & \multirow{3}{*}{ - } & \multirow{3}{*}{$10-80 \mathrm{mg} \mathrm{L}^{-1}$} & \multirow{3}{*}{10 days } & \multirow{3}{*}{$\begin{array}{l}\text { Petri dishes } \\
\text { (germination } \\
\text { test) }\end{array}$} & Germination $\% \uparrow$ at $10-40 \mathrm{mg} \mathrm{L}^{-1}$ conc. & \multirow{3}{*}{ [111] } \\
\hline & Nigella sativa $\mathrm{L}$. & & & & & & & Germination $\% \uparrow$ at $10-40 \mathrm{mg} \mathrm{L}^{-1}$ conc. & \\
\hline & $\begin{array}{l}\text { Salvia mirzayanii Rech. f. } \\
\text { \& Esfand }\end{array}$ & & & & & & & Germination $\% \uparrow$ at all conc. & \\
\hline & $\begin{array}{c}\text { Arachis hypogea L. var. } \\
\text { 'K-134' }\end{array}$ & $25 \mathrm{~nm}$ & $\mathrm{ZnO} \mathrm{NP}$ & $\begin{array}{l}100,1000 \text { and } \\
2000 \text { ppm for } \\
\text { seed priming }\end{array}$ & $\begin{array}{l}2 \text { or } 30 \mathrm{~g} / 15 \mathrm{~L} \text { for } \\
\text { foliar spraying }\end{array}$ & $\begin{array}{c}3 \mathrm{~h} \text { seed } \\
\text { priming then } \\
2 \mathrm{x} \text { foliar } \\
\text { spraying } \\
\end{array}$ & $\begin{array}{c}\text { Pot and field } \\
\text { experiment }\end{array}$ & Germination \% $\uparrow$, seedling vigour $\uparrow$ & [112] \\
\hline & Capsicum аппиит $\mathrm{L}$. & no data & $\mathrm{ZnO} \mathrm{NP}$ & $\begin{array}{l}6 \mathrm{~h} \text { seed } \\
\text { priming }\end{array}$ & $0.25,0.5$ and $0.75 \mathrm{~g}$ & 14 days & $\begin{array}{l}\text { Moistened } \\
\text { blotter paper } \\
\text { (in Petri } \\
\text { dishes) } \\
\end{array}$ & $\begin{array}{c}\text { Concentration-dependent } \uparrow \text { of seed } \\
\text { germination } \% \text {, root length } \uparrow \text {, seedling } \\
\text { length } \uparrow\end{array}$ & [113] \\
\hline
\end{tabular}


Table 1. Cont.

\begin{tabular}{|c|c|c|c|c|c|c|c|c|}
\hline Plant Name & Size of NM & $\begin{array}{l}\text { Type and } \\
\text { Chemical } \\
\text { Composition } \\
\text { of NM }{ }^{*}\end{array}$ & $\begin{array}{l}\text { Duration of } \\
\text { Pre-Cultivation }\end{array}$ & $\begin{array}{l}\text { Conc. of the NM } \\
\text { Exposure }\end{array}$ & $\begin{array}{l}\text { Time of } \\
\text { Exposure }\end{array}$ & $\begin{array}{l}\text { Growth } \\
\text { Conditions }\end{array}$ & $\begin{array}{l}\text { Main Effects on Germination and } \\
\text { Early Growth ** }\end{array}$ & Reference \\
\hline $\begin{array}{l}\text { Capsicum chinense L. var. } \\
\text { Chichen Itza }\end{array}$ & $18 \pm 8 \mathrm{~nm}$ & $\mathrm{ZnO} \mathrm{NP}$ & - & $100-500 \mathrm{mg} / \mathrm{L}$ & $\begin{array}{c}72 \mathrm{~h} \text { seed } \\
\text { priming; } 14 \\
\text { day-long } \\
\text { germination }\end{array}$ & $\begin{array}{l}\text { Petri dishes } \\
\text { (germination } \\
\text { test) }\end{array}$ & $\begin{array}{c}\text { Germination } \% \uparrow \text { with conc.; radicule } \\
\text { length } \uparrow \text { at } 300 \mathrm{mg} / \mathrm{L}\end{array}$ & [114] \\
\hline $\begin{array}{l}\text { Cucumis sativus L. } \\
\text { 'Poinsett } 76^{\prime}\end{array}$ & $8 \mathrm{~nm}$ & $\mathrm{ZnONP}$ & - & $50-1600 \mathrm{mg} \mathrm{L}^{-1}$ & $\begin{array}{l}\text { Until } 65 \% \text { of } \\
\text { the seeds were } \\
\text { germinated }\end{array}$ & $\begin{array}{l}\text { Petri dishes } \\
\text { (germination } \\
\text { test) }\end{array}$ & $\begin{array}{c}\text { Germination } \% \uparrow \text { at } 400-1600 \mathrm{mg} \mathrm{L}^{-1} \\
\text { conc. }\end{array}$ & [115] \\
\hline Vigna radiata $\mathrm{L}$. & $\sim 18 \mathrm{~nm}$ & $\mathrm{ZnO} \mathrm{NP}$ & $\begin{array}{l}\text { Seed priming } \\
\text { for } 3 \mathrm{~h}\end{array}$ & $\begin{array}{l}20,40,60,80 \\
\text { and } 100 \mathrm{mg} \mathrm{L}^{-1}\end{array}$ & $\begin{array}{l}\text { Germinating } \\
\text { for } 7 \text { days } \\
\text { after priming }\end{array}$ & $\begin{array}{l}\text { Petri dishes } \\
\text { (germination } \\
\text { test) }\end{array}$ & Germination $\% \uparrow$ & [116] \\
\hline Vigna unguiculata $\mathrm{L}$. & $30 \mathrm{~nm}$ & $\mathrm{ZnO} \mathrm{NP}$ & - & 250,500 and $750 \mathrm{ppm}$ & $\begin{array}{l}6 \mathrm{~h} \text { seed } \\
\text { treatment }\end{array}$ & $\begin{array}{c}\text { Soil (pot } \\
\text { experiment) }\end{array}$ & $\begin{array}{c}\text { Seedling length } \uparrow \text {, germination } \% \uparrow, \\
\text { seedling fresh weight } \uparrow \text { and vigour } \\
\text { index } \uparrow\end{array}$ & [117] \\
\hline
\end{tabular}

* Abbreviations: NC—nanocubes, NP—nanoparticles, NW—nanowires, LR—long nanorods, SR—short nanorods. ** $\uparrow$ indicates significant and $\uparrow n s$ indicates non-significant increase, while $\downarrow$ refers to significant decrease and $\downarrow$ ns to non-significant reduction. 
In addition to soaking, priming seeds with metallic NPs (so-called nanopriming) also seems to be effective. When Almutairi and Alharbi [107] primed watermelon and zucchini seeds for $2 \mathrm{~h}$ in Ag NP solution at low concentrations (0.5-2.0 $\left.\mathrm{g} \mathrm{L}^{-1}\right)$, then germinated the seeds at the same doses, the germination \% significantly increased, and root elongation was also promoted. Similarly, Prażak et al. [109] found that Ag NP-primed seeds of bean germinated at a higher rate compared to the control (Table 1).

Nevertheless, it may occur that metallic NP exposure has no influence on germination and early growth parameters, as it was presented in Ag NP-treated lettuce ([105]; Table 2), Pinus sylvestris and Alnus subcordata, which were germinated in Ag NP-containing soil ([118], Table 2). In the latter study, seeds were also exposed to Ag NP solutions in Petri dishes, but the germination $\%$ and seedling length were negatively affected, which suggests that the character of the growing medium is determinative in the early development of plants under NM application. 
Table 2. Mixed or no effect of metal and metal oxide NPs on seed germination and seedling growth.

\begin{tabular}{|c|c|c|c|c|c|c|c|c|c|}
\hline & Plant Name & Size of NM & $\begin{array}{l}\text { Type and } \\
\text { Chemical } \\
\text { Composition } \\
\text { of NM }{ }^{*}\end{array}$ & $\begin{array}{l}\text { Duration of } \\
\text { Pre-Cultivation }\end{array}$ & $\begin{array}{l}\text { Concentration of the } \\
\text { NM Exposure }\end{array}$ & $\begin{array}{c}\text { Time of } \\
\text { Exposure }\end{array}$ & $\begin{array}{l}\text { Growth } \\
\text { Conditions }\end{array}$ & $\begin{array}{l}\text { Main Effects on Germination and } \\
\text { Early Growth ** }\end{array}$ & Reference \\
\hline \multirow{12}{*}{ 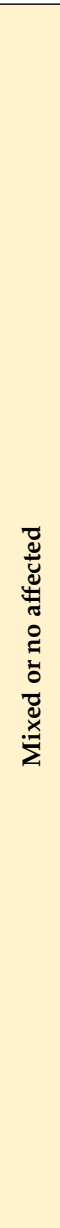 } & \multirow[b]{2}{*}{ Alnus subcordata $\mathrm{L}$. } & \multirow[b]{2}{*}{ no data } & \multirow[b]{2}{*}{$\mathrm{Ag} N P$} & - & $10-100 \mathrm{mg} \mathrm{kg}^{-1}$ (soil) & \multirow[b]{2}{*}{15 days } & $\begin{array}{c}\text { Soil (in Petri } \\
\text { dishes) }\end{array}$ & $\begin{array}{l}\text { No change of germination \% and } \\
\text { seedling length }\end{array}$ & \multirow[t]{2}{*}{ [118] } \\
\hline & & & & - & 10 and $20 \mathrm{mg} \mathrm{L}^{-1}$ & & $\begin{array}{c}\text { Petri dishes } \\
\text { (germination } \\
\text { test) }\end{array}$ & $\begin{array}{c}\text { Germination } \% \downarrow \text {; seedling length } \\
\downarrow \text { ns and } \downarrow\end{array}$ & \\
\hline & Lactuca sativa $\mathrm{L}$. & $29.2 \pm 1.1 \mathrm{~nm}$ & $\mathrm{Ag} \mathrm{NP}$ & - & $100 \mu \mathrm{g} \mathrm{mL}^{-1}$ & 7 days & $\begin{array}{l}\text { Petri dishes } \\
\text { (germination } \\
\text { test) }\end{array}$ & Germination index: no change & [105] \\
\hline & Pennisetum glaucum L. & $13 \mathrm{~nm}$ & $\mathrm{Ag} \mathrm{NP}$ & $\begin{array}{l}\text { Seed soaking } \\
\text { for } 2 \mathrm{~h} \text { in test } \\
\text { solution }\end{array}$ & 20 and $50 \mathrm{mg} \mathrm{L}^{-1}$ & 5 days & $\begin{array}{l}\text { Petri dishes } \\
\text { (germination } \\
\text { test) }\end{array}$ & $\begin{array}{l}\text { Germination } \% \uparrow \text { at higher conc.; total } \\
\text { seedling length } \downarrow \text { at higher conc. }\end{array}$ & [119] \\
\hline & \multirow[b]{2}{*}{ Pinus sylvestris $\mathrm{L}$. } & \multirow[b]{2}{*}{ no data } & \multirow[b]{2}{*}{$\mathrm{Ag} N P$} & - & $10-100 \mathrm{mg} \mathrm{kg}^{-1}$ (soil) & \multirow[b]{2}{*}{15 days } & $\begin{array}{c}\text { Soil (in Petri } \\
\text { dishes) }\end{array}$ & $\begin{array}{l}\text { No change of germination \% and } \\
\text { seedling length }\end{array}$ & \multirow[t]{2}{*}{ [118] } \\
\hline & & & & - & 10 and $20 \mathrm{mg} \mathrm{L}^{-1}$ & & $\begin{array}{l}\text { Petri dishes } \\
\text { (germination } \\
\text { test) }\end{array}$ & $\begin{array}{c}\text { Germination } \% \downarrow \text {; seedling length } \\
\downarrow \downarrow \text { ns and } \downarrow\end{array}$ & \\
\hline & $\begin{array}{l}\text { Triticum aestivum L. cv. } \\
\text { NARC-2009 }\end{array}$ & $10-20 \mathrm{~nm}$ & $\mathrm{Ag} N P$ & - & $25-150 \mathrm{ppm}$ & 7 days & $\begin{array}{c}\text { Petri dishes } \\
\text { (germination } \\
\text { test) }\end{array}$ & $\begin{array}{l}\text { Germination } \% \uparrow n s \text { and number of } \\
\text { seminal roots } \uparrow \text { at } 25-75 \text { ppm but } \downarrow \text { at } \\
\text { higher conc. }\end{array}$ & [120] \\
\hline & Zea mays L. & $20 \mathrm{~nm}$ & $\mathrm{Ag} \mathrm{NP}$ & $\begin{array}{l}2 \mathrm{~h} \text { seed } \\
\text { priming }\end{array}$ & $0.05-2.5 \mathrm{mg} \mathrm{L}^{-1}$ & $12-16$ days & $\begin{array}{c}\text { Petri dishes } \\
\text { (germination } \\
\text { test) }\end{array}$ & $\begin{array}{l}\text { No effect on germination \%; root length } \\
\qquad \text { at all conc. }\end{array}$ & [107] \\
\hline & $\begin{array}{l}\text { Cucumis sativus } \mathrm{L} \text {. } \\
\text { 'Poincett } 76^{\prime}\end{array}$ & $7 \mathrm{~nm}$ & $\mathrm{CeO}_{2} \mathrm{NP}$ & - & $500-4000 \mathrm{mg} \mathrm{L}^{-1}$ & 9 days & $\begin{array}{c}\text { Petri dishes } \\
\text { (germination } \\
\text { test) }\end{array}$ & $\begin{array}{l}\text { Germination } \% \downarrow \text { at } 2000 \mathrm{mg} \mathrm{L}^{-1} \text { conc.; } \\
\text { root and shoot length } \uparrow \text { at all conc. }\end{array}$ & [121] \\
\hline & Glycine max $\mathrm{L}$. & $7 \mathrm{~nm}$ & $\mathrm{CeO}_{2} \mathrm{NP}$ & - & $500-4000 \mathrm{mg} \mathrm{L}^{-1}$ & $\begin{array}{l}\text { Until } 65 \% \text { of } \\
\text { control roots } \\
\text { were } 5 \mathrm{~mm} \\
\quad \text { long } \\
\end{array}$ & $\begin{array}{l}\text { Petri dishes } \\
\text { (germination } \\
\text { test) }\end{array}$ & $\begin{array}{l}\text { Germination } \% \downarrow \text { at } 2000 \mathrm{mg} \mathrm{L}^{-1} \text { conc.; } \\
\quad \text { root elongation } \uparrow \text { at all conc. }\end{array}$ & {$[30]$} \\
\hline & $\begin{array}{l}\text { Medicago sativa } \mathrm{L} \text {. Mesa } \\
\text { variety }\end{array}$ & $7 \mathrm{~nm}$ & $\mathrm{CeO}_{2} \mathrm{NP}$ & - & $500-4000 \mathrm{mg} \mathrm{L}^{-1}$ & 9 days & $\begin{array}{l}\text { Petri dishes } \\
\text { (germination } \\
\text { test) }\end{array}$ & $\begin{array}{c}\text { Germination \% was not affected; root } \\
\text { length } \downarrow \text { at } 2000-4000 \mathrm{mg} \mathrm{L}^{-1} \text {, while } \\
\text { shoot length } \uparrow \text { at } 500-1000 \mathrm{mg} \mathrm{L}^{-1} \text { conc. }\end{array}$ & {$[121]$} \\
\hline & Zea mays L. & $7 \mathrm{~nm}$ & $\mathrm{CeO}_{2} \mathrm{NP}$ & - & $500-4000 \mathrm{mg} \mathrm{L}^{-1}$ & 8 days & $\begin{array}{l}\text { Petri dishes } \\
\text { (germination } \\
\text { test) }\end{array}$ & $\begin{array}{c}\text { Germination } \% \downarrow \text { at } 500-2000 \mathrm{mg} \mathrm{L}^{-1} \\
\text { root length } \uparrow \text { at } 4000 \mathrm{mg} \mathrm{L}^{-1}, \text { while } \\
\text { shoot length } \uparrow \text { at } 2000 \mathrm{mg} \mathrm{L}^{-1} \text { but } \downarrow \text { at } \\
4000 \mathrm{mg} \mathrm{L}^{-1} \text { conc. }\end{array}$ & \\
\hline
\end{tabular}


Table 2. Cont

\begin{tabular}{|c|c|c|c|c|c|c|c|c|}
\hline Plant Name & Size of NM & $\begin{array}{c}\text { Type and } \\
\text { Chemical } \\
\text { Composition } \\
\text { of NM }{ }^{*} \\
\end{array}$ & $\begin{array}{l}\text { Duration of } \\
\text { Pre-Cultivation }\end{array}$ & $\begin{array}{l}\text { Concentration of the } \\
\text { NM Exposure }\end{array}$ & $\begin{array}{l}\text { Time of } \\
\text { Exposure }\end{array}$ & $\begin{array}{l}\text { Growth } \\
\text { Conditions }\end{array}$ & $\begin{array}{l}\text { Main Effects on Germination and } \\
\text { Early Growth ** }\end{array}$ & Reference \\
\hline Brassica oleracea L. & $<50 \mathrm{~nm}$ & $\mathrm{Co}_{3} \mathrm{O}_{4}$ & - & $\begin{array}{l}\begin{array}{l}269.3-1000 \mathrm{mg} \mathrm{kg}^{-1} \\
\text { soil (DW) }\end{array}\end{array}$ & 14 days & $\begin{array}{l}\text { Germination test } \\
\text { in pot } \\
\text { experiment }\end{array}$ & $\begin{array}{l}\text { Germination } \% \uparrow \text { at } 269-350 \mathrm{mg} \mathrm{kg}^{-1} \\
\text { conc. but } \downarrow \text { ns at } 769-1000 \mathrm{mg} \mathrm{kg}^{-1} \text { conc. }\end{array}$ & [110] \\
\hline Cicer arietinum $\mathrm{L}$. & $<50 \mathrm{~nm}$ & $\mathrm{Fe}_{2} \mathrm{O}_{3} \mathrm{NP}$ & $\begin{array}{l}\text { Seed priming } \\
\quad \text { for } 2 \mathrm{~h}\end{array}$ & $10-200 \mathrm{mg} \mathrm{L}^{-1}$ & 3 days & $\begin{array}{l}\text { Petri dishes } \\
\text { (germination } \\
\text { test) }\end{array}$ & $\begin{array}{l}\text { Germination time } \downarrow \text { at all conc.; root } \\
\text { length } \downarrow \text { ns but shoot length } \uparrow \text { ns } \\
\text { concentration-dependently }\end{array}$ & [122] \\
\hline \multirow{3}{*}{$\begin{array}{l}\text { Oryza sativa L. } \\
\text { (Y Liangyou 1928) }\end{array}$} & $40-100 \mathrm{~nm}$ & $\mathrm{Fe}_{2} \mathrm{O}_{3} \mathrm{NC}$ & \multirow{3}{*}{$\begin{array}{l}\text { Seed priming } \\
\quad \text { for } 2 \mathrm{~h}\end{array}$} & \multirow{3}{*}{$5-150 \mathrm{mg} \mathrm{L}^{-1}$} & \multirow{3}{*}{$\begin{array}{l}10 \mathrm{~d} \text { after } \\
\text { priming }\end{array}$} & \multirow{3}{*}{$\begin{array}{l}\text { Petri dishes } \\
\text { (germination } \\
\text { test) }\end{array}$} & $\begin{array}{l}\text { Germination } \% \downarrow \text { ns while root length } \uparrow \\
\text { and shoot length } \uparrow \text { ns with conc. }\end{array}$ & \multirow{3}{*}{ [123] } \\
\hline & $\begin{array}{l}\text { Length: } 200-400 \\
\text { nm, diameter: } \\
10-20 \mathrm{~nm}\end{array}$ & $\mathrm{Fe}_{2} \mathrm{O}_{3} \mathrm{SR}$ & & & & & $\begin{array}{c}\text { Germination } \% \downarrow \mathrm{ns} \text {; root length } \uparrow \text { at } \\
5-50 \mathrm{mg} / \mathrm{L} \text { and } \uparrow \mathrm{ns} \text { at } 100-150 \mathrm{mg} \mathrm{L} \mathrm{L}^{-1} \\
\text { shoot length } \uparrow \text { and } \uparrow \mathrm{ns} \text { at } 5-50 \\
\text { and } 100 \mathrm{mg} \mathrm{L}^{-1}\end{array}$ & \\
\hline & $\begin{array}{l}\text { Length: } 500 \mathrm{~nm} \text {, } \\
\text { diameter: } 50 \mathrm{~nm}\end{array}$ & $\mathrm{Fe}_{2} \mathrm{O}_{3} \mathrm{LR}$ & & & & & $\begin{array}{c}\text { Germination } \% \downarrow \text { at } 5-100 \mathrm{mg} \mathrm{L}^{-1} \text {; root } \\
\text { length } \uparrow \text { with conc.; shoot length } \uparrow \text { at } \\
5-50 \mathrm{mg} \mathrm{L}^{-1}\end{array}$ & \\
\hline $\begin{array}{c}\text { Brassica napus L. var. } \\
\text { RGS003 }\end{array}$ & $20 \mathrm{~nm}$ & $\mathrm{TiO}_{2} \mathrm{NP}$ & - & $10-2000 \mathrm{mg} \mathrm{L}^{-1}$ & 7 days & $\begin{array}{l}\text { Petri dishes } \\
\text { (germination } \\
\text { test) }\end{array}$ & $\begin{array}{l}\text { No change of germination \% at } \\
100-1700 \mathrm{mg} \mathrm{L}^{-1} \text { but } \uparrow \mathrm{ns} \text { at } \\
2000 \mathrm{mg} \mathrm{L}^{-1} \text { conc.; no significant } \\
\text { changes of radicle length while } \\
\text { plumule length } \downarrow \text { at } 10-1000 \mathrm{mg} \mathrm{L}^{-1} \\
\text { and } \uparrow \mathrm{ns} \text { at higher doses }\end{array}$ & [124] \\
\hline Allium cepa L. & $21 \mathrm{~nm}$ & $\mathrm{TiO}_{2} \mathrm{NP}$ & - & $10-50 \mathrm{mg} \mathrm{L}^{-1}$ & 10 days & $\begin{array}{l}\text { Wet filter paper } \\
\text { (germination } \\
\text { test) }\end{array}$ & $\begin{array}{c}\text { Germination } \% \uparrow \mathrm{ns} \text { at } 10-40 \mathrm{mg} \mathrm{L}^{-1} \\
\text { conc.; radicle length } \uparrow \mathrm{ns} \text { at } \\
10-30 \mathrm{mg} \mathrm{L}^{-1} \text { but } \downarrow \text { at higher doses, } \\
\text { while shoot length } \uparrow\end{array}$ & [125] \\
\hline Carum copticum $\mathrm{L}$. & $10-25 \mathrm{~nm}$ & $\mathrm{TiO}_{2} \mathrm{NP}$ & - & $10-80 \mathrm{mg} \mathrm{L}^{-1}$ & 10 days & $\begin{array}{l}\text { Petri dishes } \\
\text { (germination } \\
\text { test) }\end{array}$ & $\begin{array}{l}\text { Germination } \% \uparrow \text { at } 10-20 \mathrm{mg} \mathrm{L}^{-1} \\
\text { but } \downarrow \text { at higher conc. }\end{array}$ & [111] \\
\hline $\begin{array}{l}\text { Oryza sativa L. } \\
(\text { Y Liangyou 1928) }\end{array}$ & $20 \mathrm{~nm}$ & $\mathrm{TiO}_{2} \mathrm{NP}$ & - & $5-150 \mathrm{mg} \mathrm{L}^{-1}$ & $\begin{array}{l}\text { Seed priming } \\
\text { for } 2 \mathrm{~h} \text { then } \\
\text { cultivation } \\
\text { for } 10 \mathrm{~d}\end{array}$ & $\begin{array}{l}\text { Petri dishes } \\
\text { (germination } \\
\text { test) }\end{array}$ & $\begin{array}{c}\text { Germination } \% \downarrow \mathrm{ns} \text {; root length } \uparrow \text { at } \\
\text { 5-10,50 and } 100 \mathrm{mg} \mathrm{L}^{-1} \text {; shoot length } \uparrow \\
\text { at } 150 \mathrm{mg} \mathrm{L}^{-1}\end{array}$ & [123] \\
\hline Sinapis alba L. & $10-25 \mathrm{~nm}$ & $\mathrm{TiO}_{2} \mathrm{NP}$ & - & $10-80 \mathrm{mg} \mathrm{L}^{-1}$ & $10 \mathrm{~d}$ & $\begin{array}{l}\text { Petri dishes } \\
\text { (germination } \\
\text { test) }\end{array}$ & $\begin{array}{l}\text { Germination } \% \uparrow \text { at } 10-20 \mathrm{mg} \mathrm{L}^{-1} \\
\text { but } \downarrow \text { at higher conc. }\end{array}$ & [111] \\
\hline Allium cepa $\mathrm{L}$. & $20 \mathrm{~nm}$ & $\mathrm{ZnONP}$ & - & $10-40 \mathrm{mg} \mathrm{L}^{-1}$ & 10 days & $\begin{array}{l}\text { Wet filter paper } \\
\text { (germination } \\
\text { test) }\end{array}$ & $\begin{array}{l}\text { Germination \% and seedling growth } \\
\uparrow \text { ns at lower conc. }\end{array}$ & [126] \\
\hline
\end{tabular}


Table 2. Cont

\begin{tabular}{|c|c|c|c|c|c|c|c|c|}
\hline Plant Name & Size of NM & $\begin{array}{c}\text { Type and } \\
\text { Chemical } \\
\text { Composition } \\
\text { of NM }{ }^{*}\end{array}$ & $\begin{array}{l}\text { Duration of } \\
\text { Pre-Cultivation }\end{array}$ & $\begin{array}{l}\text { Concentration of the } \\
\text { NM Exposure }\end{array}$ & $\begin{array}{c}\text { Time of } \\
\text { Exposure }\end{array}$ & $\begin{array}{l}\text { Growth } \\
\text { Conditions }\end{array}$ & $\begin{array}{l}\text { Main Effects on Germination and } \\
\text { Early Growth ** }\end{array}$ & Reference \\
\hline Avena sativa $\mathrm{L}$. & no data & $\mathrm{ZnO} \mathrm{NP}$ & - & $\begin{array}{c}750,1000 \text { and } \\
1250 \mathrm{mg} \mathrm{kg}^{-1} \text { seed }\end{array}$ & $\begin{array}{c}10 \mathrm{~min} \\
\text { priming }\end{array}$ & $\begin{array}{l}\text { Wet paper and } \\
\text { field } \\
\text { experiment }\end{array}$ & $\begin{array}{c}\text { Germination \%, seedling vigour and } \\
\text { yield } \uparrow \text { at low conc., root and shoot } \\
\text { length } \downarrow \text { at higher doses }\end{array}$ & [127] \\
\hline $\begin{array}{l}\text { Brassica juncea L. Czern. } \\
\text { cv. Negro Caballo }\end{array}$ & \multirow{2}{*}{$45 \mathrm{~nm}$} & \multirow{2}{*}{$\mathrm{ZnO} \mathrm{NP}$} & - & \multirow{2}{*}{25 and $100 \mathrm{mg} \mathrm{L}^{-1}$} & \multirow{2}{*}{5 days } & \multirow{2}{*}{$\begin{array}{l}\text { Petri dishes } \\
\text { (germination } \\
\text { test) }\end{array}$} & \multirow{2}{*}{$\begin{array}{c}\text { Primary root length } \uparrow \text { at } 25 \mathrm{mg} \mathrm{L}^{-1} \text { but } \\
\downarrow \text { at } 100 \mathrm{mg} \mathrm{L}^{-1} \text { conc. }\end{array}$} & \multirow{2}{*}{ [32] } \\
\hline $\begin{array}{l}\text { Brassica napus L. cv. GK } \\
\text { Gabriella }\end{array}$ & & & - & & & & & \\
\hline Glycine $\max \mathrm{L}$. & $8 \mathrm{~nm}$ & $\mathrm{ZnO} \mathrm{NP}$ & - & $500-4000 \mathrm{mg} \mathrm{L}^{-1}$ & $\begin{array}{l}\text { Until } 65 \% \text { of } \\
\text { control roots } \\
\text { were } 5 \mathrm{~mm} \\
\text { long }\end{array}$ & $\begin{array}{c}\text { Petri dishes } \\
\text { with wet } \\
\text { filter paper } \\
\text { (germination } \\
\text { test) }\end{array}$ & $\begin{array}{l}\text { Germination was not affected; root } \\
\text { elongation } \uparrow \text { at } 500 \mathrm{mg} \mathrm{L}^{-1} \text { but } \downarrow \text { at } \\
2000 \mathrm{mg} \mathrm{L}^{-1} \mathrm{ZnO} \mathrm{NP}\end{array}$ & [30] \\
\hline Hordeum vulgare $\mathrm{L}$. & $30 \mathrm{~nm}$ & $\mathrm{ZnO} \mathrm{NP}$ & - & $\begin{array}{c}5,10,20,40 \\
\text { and } 80 \mathrm{mg} \mathrm{kg}^{-1}\end{array}$ & $\begin{array}{c}7 \text { days } \\
\text { germination } \\
\text { then } 21 \text { days } \\
\text { cultivation }\end{array}$ & $\begin{array}{c}\text { Petri dishes } \\
\text { (germination) } \\
\text { then pot } \\
\text { experiment }\end{array}$ & $\begin{array}{l}\text { No effect on seed germination } \\
\text { and root elongation }\end{array}$ & [128] \\
\hline Oryza sativa L. & no data & $\mathrm{ZnO} \mathrm{NP}$ & 1-3 days & $10-1000 \mathrm{mg} \mathrm{L}^{-1}$ & 7 days & $\begin{array}{l}\text { Moistened } \\
\text { filter paper }\end{array}$ & No change in germination $\%$ & [129] \\
\hline Oryza sativa L. & $\leq 50 \mathrm{~nm}$ & $\mathrm{ZnO} \mathrm{NP}$ & $2 \mathrm{~h}$ & $10-500 \mathrm{mg} \mathrm{L}^{-1}$ & 5-12 days & $\begin{array}{l}\text { Petri dishes } \\
\text { (filter paper or } \\
\text { soil) }\end{array}$ & No change of germination $\%$ & [130] \\
\hline Pennisetum glaucum $\mathrm{L}$. & $<50 \mathrm{~nm}$ & $\mathrm{ZnO} \mathrm{NP}$ & - & $100-1000 \mathrm{mg} \mathrm{L}^{-1}$ & 7 days & $\begin{array}{l}\text { Petri dishes } \\
\text { (germination } \\
\text { test) }\end{array}$ & $\begin{array}{l}\text { Germination } \% \downarrow \text {; root length } \uparrow \\
\text { but } \downarrow \text { at } 500-1000 \mathrm{mg} \mathrm{L}^{-1} \text { conc.; } \\
\text { shoot length } \uparrow \mathrm{ns} \text { and } \downarrow \text { ns }\end{array}$ & [131] \\
\hline $\begin{array}{l}\text { Phaseolus vulgaris L. var. } \\
\text { red hawk kidney }\end{array}$ & 93.8 or $84.1 \mathrm{~nm}$ & $\mathrm{ZnO} \mathrm{NP}$ & - & $\begin{array}{l}\begin{array}{l}62.5-500 \mathrm{mg} \mathrm{kg}^{-1} \\
\text { (soil) }\end{array} \\
\text { (s) }\end{array}$ & 45 days & $\begin{array}{c}\text { Soil (pot } \\
\text { experiment) }\end{array}$ & No effect on germination $\%$ & [132] \\
\hline $\begin{array}{l}\text { Solanum lycopersicum L. } \\
\text { hybr. 'tomato cherry } \\
\text { super sweet } 100^{\prime}\end{array}$ & $28 \pm 0.7 \mathrm{~nm}$ & $\mathrm{ZnO} \mathrm{NP}$ & $\begin{array}{l}\text { Seed priming } \\
\quad \text { for } 1 \mathrm{~h}\end{array}$ & $10-1000 \mathrm{mg} \mathrm{L}^{-1}$ & 5 days & $\begin{array}{l}\text { Petri dishes } \\
\text { (germination } \\
\text { test) }\end{array}$ & $\begin{array}{l}\text { No change of germination \% at } \\
10-750 \mathrm{mg} \mathrm{L}^{-1} \text { but } \downarrow \text { at } \\
1000 \mathrm{mg} \mathrm{L}^{-1} \text { conc. }\end{array}$ & [133] \\
\hline Trifolium alexandrium $\mathrm{L}$. & no data & $\mathrm{ZnO} \mathrm{NP}$ & $\begin{array}{l}10 \mathrm{~min} \\
\text { priming }\end{array}$ & $\begin{array}{c}750,1000 \text { and } \\
1250 \mathrm{mg} \mathrm{kg}^{-1} \text { seed }\end{array}$ & no data & $\begin{array}{l}\text { Wet paper } \\
\text { and field } \\
\text { experiment }\end{array}$ & $\begin{array}{c}\text { Germination } \% \text {, seedling vigour and } \\
\text { yield } \uparrow \text { at low conc., root and shoot } \\
\text { length } \downarrow \text { at higher doses }\end{array}$ & [127] \\
\hline
\end{tabular}

Abbreviations: $\mathrm{NC}-$ nanocubes, $\mathrm{NP}-$ nanoparticles, $\mathrm{NW}$-nanowires,
while $\downarrow$ refers to significant decrease and $\downarrow$ ns to non-significant reduction. 
Ag NPs, which are mainly used due to their antibacterial activity in medical practice, can be detrimental for germinating seeds and developing plantlets [134]. In the study by Geisler-Lee et al. [134], Arabidopsis seeds were germinated in the presence of 20, 40 or $80 \mathrm{~nm}$-sized Ag NPs at 66.84, 133.68, 267.36 and $534.72 \mathrm{mg} \mathrm{L}^{-1}$ concentrations, then the elongation rate of the roots was determined. The uptake of Ag NPs by the roots was clearly exhibited, moreover, Ag NP toxicity was shown to be size and concentration dependent. While $80 \mathrm{~nm}$-sized Ag NPs were only deteriorative at higher doses, those of 20 and $40 \mathrm{~nm}$ caused severe growth inhibition of the root. All the Ag NP-treated roots had typical brownish root apices, and the NPs were localized in border cells, root cap, columella and columella initials. The researchers supposed that Ag NPs were apoplastically transported through the root tissues. The inhibitory effect of Ag NPs on the germination index was also seen in the case of cucumber ([105]; Table 3). 
Table 3. Negative effects of metal and metal oxide NPs on seed germination and seedling growth.

\begin{tabular}{|c|c|c|c|c|c|c|c|c|c|}
\hline & Plant Name & Size of NM & $\begin{array}{c}\text { Type and } \\
\text { Chemical } \\
\text { Composition } \\
\text { of NM }\end{array}$ & $\begin{array}{l}\text { Duration of } \\
\text { Pre-Cultivation }\end{array}$ & $\begin{array}{l}\text { Concentration of the } \\
\text { NM Exposure }\end{array}$ & $\begin{array}{c}\text { Time of } \\
\text { Exposure }\end{array}$ & $\begin{array}{l}\text { Growth } \\
\text { Conditions }\end{array}$ & $\begin{array}{l}\text { Main Effects on Germination and } \\
\text { Early Growth ** }\end{array}$ & Reference \\
\hline \multirow{15}{*}{ 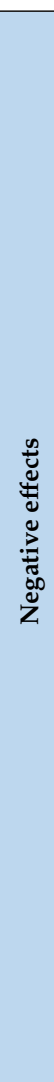 } & Cucumis sativus $\mathrm{L}$. & $29.2 \pm 1.1 \mathrm{~nm}$ & $\mathrm{Ag} \mathrm{NP}$ & - & $100 \mu \mathrm{g} \mathrm{mL}^{-1}$ & 7 days & $\begin{array}{c}\text { Petri dishes } \\
\text { (germination } \\
\text { test) }\end{array}$ & Germination index $\downarrow$ & [105] \\
\hline & Lolium multiflorum L. & $35 \pm 7 \mathrm{~nm}$ & $\mathrm{Ag} \mathrm{NP}$ & \multirow{2}{*}{ - } & \multirow{2}{*}{$10 \mathrm{ppm}$} & \multirow{2}{*}{6 days } & \multirow{2}{*}{$\begin{array}{l}\text { Petri dishes } \\
\text { (germination } \\
\text { test) }\end{array}$} & $\begin{array}{l}\text { Root length } \downarrow \text { ns but physical separation } \\
\text { from NPs caused } \uparrow\end{array}$ & \multirow{2}{*}{ [108] } \\
\hline & Lolium multiflorum L. & $44 \pm 7 \mathrm{~nm}$ & $\mathrm{Ag} \mathrm{NC}$ & & & & & $\begin{array}{l}\text { Root length } \downarrow \text { ns but physical separation } \\
\text { from NCs caused } \uparrow\end{array}$ & \\
\hline & Phaseolus radiatus $\mathrm{L}$. & no data & $\mathrm{Cu} \mathrm{NP}$ & \multirow{2}{*}{$24 \mathrm{~h}$} & \multirow{2}{*}{$200-1000 \mathrm{mg} \mathrm{L}^{-1}$} & \multirow{2}{*}{$48 \mathrm{~h}$} & \multirow{2}{*}{$\begin{array}{l}\text { Petri dishes } \\
\text { (germination } \\
\text { test, agar) }\end{array}$} & $\begin{array}{l}\text { Seedling growth and root growth } \downarrow \\
\text { concentration-dependently }\end{array}$ & \multirow{2}{*}{ [135] } \\
\hline & $\begin{array}{l}\text { Triticum aestivum ssp. } \\
\text { aestivum }\end{array}$ & no data & $\mathrm{Cu} \mathrm{NP}$ & & & & & $\begin{array}{l}\text { Seedling growth and root growth } \downarrow \\
\text { concentration-dependently }\end{array}$ & \\
\hline & $\begin{array}{l}\text { Solanum lycopersicum L. } \\
\text { 'Pomodoro' }\end{array}$ & $7 \mathrm{~nm}$ & $\mathrm{CeO}_{2} \mathrm{NP}$ & - & $500-4000 \mathrm{mg} \mathrm{L}^{-1}$ & 6 days & $\begin{array}{c}\text { Petri dishes } \\
\text { (germination } \\
\text { test) }\end{array}$ & $\begin{array}{l}\text { Germination } \% \downarrow \text { ns and } \downarrow \text {; root } \\
\text { elongation } \downarrow \text { at } 1000-4000 \mathrm{mg} / \mathrm{L} \text { conc. }\end{array}$ & [121] \\
\hline & Avena sativa $\mathrm{L}$. & \multirow[b]{2}{*}{$<50 \mathrm{~nm}$} & \multirow[b]{2}{*}{$\mathrm{Co}_{3} \mathrm{O}_{4} \mathrm{NP}$} & \multirow[b]{2}{*}{ - } & \multirow{2}{*}{$\begin{array}{l}269.3-1000 \mathrm{mg} \mathrm{kg}^{-1} \\
\text { soil }(\mathrm{DW})\end{array}$} & \multirow[b]{2}{*}{14 days } & \multirow{2}{*}{$\begin{array}{c}\text { Germination } \\
\text { test in pot } \\
\text { experiment }\end{array}$} & Germination $\% \downarrow$ ns at higher conc. & \multirow[b]{2}{*}{ [110] } \\
\hline & Solanum lycopersicum $\mathrm{L}$. & & & & & & & $\begin{array}{l}\text { Germination } \% \downarrow \text { ns but } \downarrow \\
\text { at } 1000 \mathrm{mg} / \mathrm{kg} \text { conc. }\end{array}$ & \\
\hline & $\begin{array}{l}\text { Oryza sativa L. Jijing } \\
\text { No.6. }\end{array}$ & $<50 \mathrm{~nm}$ & $\mathrm{CuONP}$ & $2 \mathrm{~h}$ priming & $25-2000 \mathrm{mg} \mathrm{L}^{-1}$ & $\begin{array}{l}\text { Germination } \\
\text { for } 5 \text { days } \\
\text { after priming }\end{array}$ & $\begin{array}{l}\text { Petri dishes } \\
\text { (germination } \\
\text { test) }\end{array}$ & Root length $\downarrow$ at all conc. & [136] \\
\hline & $\begin{array}{l}\text { Zea mays L. Zhengdan } \\
\text { No. } 958 .\end{array}$ & $<50 \mathrm{~nm}$ & $\mathrm{CuONP}$ & $2 \mathrm{~h}$ priming & $25-2000 \mathrm{mg} \mathrm{L}^{-1}$ & $\begin{array}{l}\text { Germination } \\
\text { for } 7 \text { days } \\
\text { after priming }\end{array}$ & $\begin{array}{c}\text { Petri dishes } \\
\text { (germination } \\
\text { test) }\end{array}$ & Root length $\downarrow$ at all conc. & [136] \\
\hline & $\begin{array}{l}\text { Arabidopsis thaliana } \\
\text { 'Col- } 0 \text { ' }\end{array}$ & $<50 \mathrm{~nm}$ & $\mathrm{Fe}_{3} \mathrm{O}_{4} \mathrm{NP}$ & $\begin{array}{c}5 \text { days at } 4^{\circ} \mathrm{C} \\
\text { (in dark) }\end{array}$ & $\begin{array}{l}400,2000 \text { and } \\
4000 \mathrm{mg} \mathrm{L}^{-1}\end{array}$ & 18 days & $\begin{array}{l}1 / 2 \mathrm{MS} \\
\text { medium }\end{array}$ & $\begin{array}{l}\text { Seed germination } \% \downarrow \text { ns at } 400 \text { and } \\
2000 \mathrm{mg} \mathrm{L}^{-1} \text {; root elongation } \downarrow\end{array}$ & [137] \\
\hline & Cucumis sativus $\mathrm{L}$. & \multirow{2}{*}{$7.57 \pm 5.6 \mathrm{~nm}$} & \multirow{2}{*}{$\mathrm{Fe}_{3} \mathrm{O}_{4} \mathrm{NP}$} & \multirow{2}{*}{-} & \multirow{2}{*}{$116 \mu \mathrm{g} \mathrm{mL}^{-1}$} & \multirow{2}{*}{7 days } & \multirow{2}{*}{$\begin{array}{c}\text { Petri dishes } \\
\text { (germination } \\
\text { test) }\end{array}$} & Germination index $\downarrow$ & \multirow[t]{2}{*}{ [105] } \\
\hline & Lactuca sativa $\mathrm{L}$. & & & & & & & Germination index $\downarrow$ & \\
\hline & Vicia narbonensis $\mathrm{L}$. & \multirow{2}{*}{$<100 \mathrm{~nm}$} & \multirow{2}{*}{$\mathrm{TiO}_{2} \mathrm{NP}$} & \multirow{2}{*}{$\begin{array}{l}\text { Soaking seed } \\
\text { for } 24 \mathrm{~h} \text { in the } \\
\text { test solutions }\end{array}$} & \multirow{2}{*}{$0.2-4.0 \%$} & \multirow{2}{*}{$72 \mathrm{~h}$} & \multirow{2}{*}{$\begin{array}{l}\text { Petri dishes } \\
\text { (germination } \\
\text { test) }\end{array}$} & Germination $\% \downarrow$ ns; root elongation $\downarrow$ & \multirow[t]{2}{*}{ [138] } \\
\hline & Zea mays L. & & & & & & & $\begin{array}{l}\text { ns and } \downarrow \text {, chromosomal aberration } \\
\text { index in root tip meristem } \uparrow \text { with conc. }\end{array}$ & \\
\hline
\end{tabular}


Table 3. Cont

\begin{tabular}{|c|c|c|c|c|c|c|c|c|}
\hline Plant Name & Size of NM & $\begin{array}{l}\text { Type and } \\
\text { Chemical } \\
\text { Composition } \\
\text { of NM }{ }^{*}\end{array}$ & $\begin{array}{l}\text { Duration of } \\
\text { Pre-Cultivation }\end{array}$ & $\begin{array}{l}\text { Concentration of the } \\
\text { NM Exposure }\end{array}$ & $\begin{array}{l}\text { Time of } \\
\text { Exposure }\end{array}$ & $\begin{array}{l}\text { Growth } \\
\text { Conditions }\end{array}$ & $\begin{array}{l}\text { Main Effects on Germination and } \\
\text { Early Growth ** }\end{array}$ & Reference \\
\hline $\begin{array}{l}\text { Arabidopsis thaliana } \\
\text { 'Col- } 0 \text { ' }\end{array}$ & $\sim 44 \mathrm{~nm}$ & $\mathrm{ZnO} \mathrm{NP}$ & $\begin{array}{c}5 \text { days at } 4{ }^{\circ} \mathrm{C} \\
\text { (in dark) }\end{array}$ & $\begin{array}{l}400,2000 \text { and } \\
4000 \mathrm{mg} \mathrm{L}^{-1}\end{array}$ & 18 days & $\begin{array}{l}1 / 2 \mathrm{MS} \\
\text { medium }\end{array}$ & $\begin{array}{c}\text { Seed germination } \% \downarrow \text { and root } \\
\text { elongation } \downarrow\end{array}$ & [137] \\
\hline $\begin{array}{l}\text { Brassica napus L. cv. } \\
\quad \text { Hayola } 401\end{array}$ & $<50 \mathrm{~nm}$ & $\mathrm{ZnO} \mathrm{NP}$ & - & $5-500 \mathrm{mg} \mathrm{L}^{-1}$ & 6 days & $\begin{array}{l}\text { Petri dishes } \\
\text { (germination } \\
\text { test) }\end{array}$ & Germination $\% \downarrow \mathrm{ns}$ & [139] \\
\hline Cucumis satious $\mathrm{L}$. & $\leq 50 \mathrm{~nm}$ & $\mathrm{ZnO} \mathrm{NP}$ & $2 \mathrm{~h}$ & $10-500 \mathrm{mg} / \mathrm{L}$ & 5-12 days & $\begin{array}{l}\text { Petri dishes } \\
\text { (filter paper or } \\
\text { soil) }\end{array}$ & Germination $\% \downarrow$ ns & [130] \\
\hline $\begin{array}{l}\text { Oryza sativa L. Jijing No. } \\
6 .\end{array}$ & $<50 \mathrm{~nm}$ & $\mathrm{ZnONP}$ & $2 \mathrm{~h}$ priming & $25-2000 \mathrm{mg} / \mathrm{L}$ & $\begin{array}{l}\text { Germination } \\
\text { for } 5 \text { days } \\
\text { after priming }\end{array}$ & $\begin{array}{l}\text { Petri dishes } \\
\text { (germination } \\
\text { test) }\end{array}$ & $\begin{array}{c}\text { Germination \% was not affected at } \\
2000 \mathrm{mg} \mathrm{L}^{-1} \text { conc.; root length } \downarrow \text { at } \\
100-2000 \mathrm{mg} \mathrm{L}^{-1}\end{array}$ & [136] \\
\hline $\begin{array}{l}\text { Solanum lycopersicum L. } \\
\text { 'Roma FV' }\end{array}$ & $8 \mathrm{~nm}$ & $\mathrm{ZnONP}$ & - & $50-1600 \mathrm{mg} \mathrm{L}^{-1}$ & $\begin{array}{l}\text { Until } 65 \% \text { of } \\
\text { the seeds were } \\
\text { germinated }\end{array}$ & $\begin{array}{l}\text { Petri dishes } \\
\text { (germination } \\
\text { test) }\end{array}$ & $\begin{array}{l}\text { Germination } \% \downarrow \text { at } \\
800-1600 \mathrm{mg} \mathrm{L}^{-1} \text { conc. }\end{array}$ & [115] \\
\hline Solanum lycopersicum $\mathrm{L}$. & \multirow[b]{2}{*}{$<50 \mathrm{~nm}$} & \multirow[b]{2}{*}{$\mathrm{ZnONP}$} & \multirow[b]{2}{*}{-} & \multirow[b]{2}{*}{$100-1000 \mathrm{mg} \mathrm{L}^{-1}$} & \multirow[b]{2}{*}{7 days } & \multirow{2}{*}{$\begin{array}{l}\text { Petri dishes } \\
\text { (germination } \\
\text { test) }\end{array}$} & Germination $\% \downarrow$ at $750-1000 \mathrm{mg} \mathrm{L}^{-1}$ & \multirow[b]{2}{*}{ [131] } \\
\hline Triticum aestivum $\mathrm{L}$. & & & & & & & $\begin{array}{l}\text { Germination \% } \downarrow \text { from } \\
250 \mathrm{mg} \mathrm{L}^{-1} \mathrm{ZnO} \mathrm{NP}\end{array}$ & \\
\hline $\begin{array}{c}\text { Triticum aestivum } \mathrm{L} . \\
\text { Vigna radiata } \mathrm{L} .\end{array}$ & $\leq 50 \mathrm{~nm}$ & $\mathrm{ZnONP}$ & $2 \mathrm{~h}$ & $10-500 \mathrm{mg} \mathrm{L}^{-1}$ & 5-12 days & $\begin{array}{l}\text { Petri dishes } \\
\text { (filter paper or } \\
\text { soil) }\end{array}$ & Germination $\% \downarrow$ ns & [130] \\
\hline $\begin{array}{l}\text { Zea mays L. Zhengdan } \\
\text { No. } 958 .\end{array}$ & $<50 \mathrm{~nm}$ & $\mathrm{ZnO} \mathrm{NP}$ & $2 \mathrm{~h}$ priming & $25-2000 \mathrm{mg} \mathrm{L}^{-1}$ & $\begin{array}{l}\text { Germination } \\
\text { for } 7 \text { days } \\
\text { after priming }\end{array}$ & $\begin{array}{l}\text { Petri dishes } \\
\text { (germination } \\
\text { test) }\end{array}$ & $\begin{array}{c}\text { Germination \% was not affected at } \\
2000 \mathrm{mg} \mathrm{L}^{-1} \text { conc.; root length } \downarrow \text { at } \\
500-2000 \mathrm{mg} \mathrm{L}^{-1}\end{array}$ & [136] \\
\hline
\end{tabular}


Though copper $(\mathrm{Cu})$ is an essential element for plant growth and has a key role in the photosynthesis, we have a few data about the impacts of $\mathrm{Cu}$ NPs on seed germination and early seedling development. Lee et al. [135] tested the effect of $\mathrm{Cu}$ NPs on mung bean and wheat at relatively high doses (200-1000 $\left.\mathrm{mg} \mathrm{L}^{-1}\right)$ and both seedling growth and root growth were shown to decrease in a concentration-dependent manner (Table 3). Much lower doses $\left(0.2-1 \mathrm{mg} \mathrm{L}^{-1}\right.$ ) of Cu NPs were used in the study of Hafeez et al. [140] in the germination test executed on wheat (Pakistani wheat cultivar Millat-2011) and only the highest concentration evoked a lower germination percentage. In the experiment of Zuverza-Mena et al. [141], cilantro (Coriandrum sativum) seeds (fruits) were germinated in the soil containing 20 and $80 \mathrm{mg} \mathrm{kg}^{-1} \mathrm{Cu}$ NPs. Germination \% was negatively influenced only at the lower concentration, root elongation seemed to be not affected, while shoot length significantly decreased at the higher dose of $\mathrm{Cu}$ NPs. From these results, it seems that not only the concentration but the type of growth medium may affect the outcome of a study applying various metallic NPs.

\subsubsection{Metal Oxide NPs}

Among metallic oxide NMs, ZnO NP is one of the most widely used engineered NMs in the industries, e.g., cosmetics or therapeutics, due to its anticancer and antimicrobial effect (reviewed by $[37,142]$ ). It has been documented in numerous plant species (including crops) that the optimal amount of ZnO NPs might have a positive effect on seed germination and seedling growth, also depending on particle size. In germination tests executed in Petri dishes, the germination efficiency expressed as germination \% seemed to be induced by $\mathrm{ZnO}$ NP application in the case of cucumber [115], mung bean [116], and chili pepper [114]. In addition, ZnO NP application proved to be beneficial in pot and field experiments as well ([112,117]; Table 1).

At the same time, ZnO NP exposure may also have no effect on germination \%, as it was demonstrated in soybean [30] and rice [130] germinated in Petri dishes, in barley [128], bean [132] and in scots pine [118] cultivated in pot experiments (Table 2). Nevertheless, the dual aspects of ZnO NPs were exhibited in case of onion [126], oat [127] and Brassica species [32] when germination \% and/or primary root elongation were promoted by a low dosage of ZnO NPs but those were inhibited by higher concentrations (Table 2). In the latter study [32], the authors presumed that the $\mathrm{ZnO}$ NPs of small size $(8 \mathrm{~nm})$ could enter the root cells and be at least partially biotransformed into ionic form. Moreover, ZnO NPs might cause the imbalance of ROS and/or RNS (reactive nitrogen species), resulting in the lower viability of root meristem cells and inhibited root elongation, especially in the sensitive species.

From many studies, it has emerged that ZnO NPs can act as a stressor, inducing a reduction in both seed germination as well as the growth of the radicle and the plumule. Not significant but diminished germination \% was observed under $\mathrm{ZnO} \mathrm{NP}$ exposure in rapeseed [139], wheat and cucumber [130]. Severe inhibitory effects of ZnO NPs were displayed in the case of Arabidopsis at all doses [137], while tomato proved to be sensitive at higher $\left(>750-800 \mathrm{mg} \mathrm{L}^{-1}\right) \mathrm{ZnO} N \mathrm{~N}$ concentrations ([115,131]; Table 3).

According to these findings, we may presume that there might be a correlation between the inhibition of root elongation and mitotic disorders in the root tip cells, accompanied by the increase in root diameter [143] or lateral root number [144], which suggests the potential reorientation of root cells like in so-called stress-induced morphogenic responses (SIMR, [145]). Moreover, the tolerance against $\mathrm{ZnO}$ NP exposure appears to be related to the constitution of the root cell wall, namely the increase in the amount of lignin, suberin, pectin or callose, as it was presented by Molnár et al. [43].

$\mathrm{TiO}_{2} \mathrm{NPs}$, which are mainly applied in the cosmetics and paint industry, can differently influence the seed germination process of plants. Its promotive impact was established in the germination tests of Alyssum homolocarpum, Nigella sativa and Salvia mirzayanii ([111], Table 1). In other experiments executed on onion [125], white mustard [111] and rice [123], lower concentrations (10-100 $\mathrm{mg} \mathrm{L}^{-1}$, depending on plant species) were found to be stimulative for germination or root growth while higher doses proved to be toxic (Table 2). Absolutely deleterious aspects of $\mathrm{TiO}_{2} \mathrm{NPs}$ were presented in Vicia narbonensis and maize seedlings, where root growth inhibition induced by NPs was due to chromosomal aberrations in root tip meristem cells ([138], Table 3). 
Adhikari et al. [146] reported that the germination itself of soybean and chickpea seeds exposed to $\mathrm{CuONPs}$ at 5 up to $2000 \mathrm{mg} \mathrm{L}^{-1}(<50 \mathrm{~nm})$ was not inhibited probably due to the seed coat but later toxic effects were exhibited in the root elongation in the case of both crops. The roots of both soybean and chickpea seedlings were remarkably shorter compared to the control from $600 \mathrm{mg} \mathrm{L}^{-1}$. The negative effects of $\mathrm{CuO}$ NPs were also proved in graminaceous crops, rice and maize ([136], Table 3).

$\mathrm{Fe}_{3} \mathrm{O}_{4} \mathrm{NPs}$, which are preferred in biomedicine (e.g., cancer nanotherapy, [39]), were exhibited to have a slight beneficial effect in case of chickpea, since NP-application improved the germination time at all concentrations and induced better shoot growth (Table 2, [122]). At the same time, in the study of Barrena et al. [105], $\mathrm{Fe}_{3} \mathrm{O}_{4}$ NPs proved to inhibit the germination of cucumber and lettuce seeds and reduce the elongation of the primary roots, which was probably due to the small size $(7.57 \pm 5.6 \mathrm{~nm})$ of the NPs and their potentially easier uptake by the developing roots. Furthermore, excess iron (Fe) can be highly reactive and toxic because of ROS overproduction via the Fenton reaction [147].

\subsubsection{Metal Containing Quantum Dots}

Quantum dots are photoreactive nanocrystal consisting of a core and a shell. The optical characteristics of the quantum dot is determined by the reactive core, which can be made with metals and semiconductors, for example cadmium selenide (CdSe), zinc selenide ( $\mathrm{ZnSe}$ ), etc. To date, the use of these NMs has been limited to the medical field and new industrial applications such as solar panels or telecommunications [148]. However, in biological systems, QDs have already been used for biolabeling in animal cells [99], and we have only a few data about the impact of these nanocrystals on early plant development. When rice seeds were treated with water-soluble MPA-linked CdSe quantum dots (QDs) at three different concentrations $\left(0.25 \mathrm{~mL}\right.$ QDs+1.25 mL H $\mathrm{H}_{2} \mathrm{O}, 0.5 \mathrm{~mL}$ QDs+1 mL $\mathrm{H}_{2} \mathrm{O}$ and $1 \mathrm{~mL}$ QDs $+0.5 \mathrm{~mL} \mathrm{H}_{2} \mathrm{O}$ ), the researchers found that there were higher concentrations that blocked germination while the very low concentration of QD solution had no inhibitory effect [149]. Das et al. [150] investigated the effect of the ultra-small size $(<5 \mathrm{~nm}) \mathrm{N}$-acetyl cysteine (NAC)-coated core-shell CdS: Mn/ZnS QDots (NAC-QDs) on the seed germination and seedling growth of garden pea. The seeds were soaked for $24 \mathrm{~h}$ in different concentrations of NAC-QD $(2,5,10,20,40,60,80$ and $100 \mu \mathrm{g} \mathrm{mL}^{-1}$ ), then germinated in Petri dishes containing the test solutions for 5 days. Germination \% was similar to the control up to $40 \mu \mathrm{g} \mathrm{mL} \mathrm{L}^{-1}$, but at higher doses, significant decrement occurred with visible signs of root growth inhibition and brownish root tips.

However, the investigations of Koo et al. [99] were executed on adult Arabidopsis plants, and their results confirmed that the effects of QDs do not only depend on the size and composition but the coat characteristics also have influence on their stability, uptake, and translocation, even in germinating plants.

\subsection{The Effect of Dendrimers on Seed Germination}

Dendrimers are hyperbranched nanoscale polymers with a uniform size, described molecular weight, large inner cavities and a high number of surface groups that make them particularly tunable in terms of solution chemistry. Dendritic macromolecules tend to linearly increase in diameter and adopt a more globular shape with increasing dendrimer generation. For this reason, dendrimers have become an ideal way to clearly study the effects of polymer size, charge, and composition on biologically relevant properties, cytotoxicity, blood plasma retention time internalization, biodistribution, and filtration [151].

The most common dendrimer scaffold that is commercially available is prepared from polyamidoamine (PAMAM) dendrimers [152]. Dendrimers act as a platform for nitric oxide (NO) transport and delivery, but their application in agriculture is still not explored [153,154]. The usage of dendrimers is widespread, ranging from biology to material sciences [155]. However, NAAS (National Academy of Agricultural Sciences) [156], showed that nanoscale carriers in polymers and dendrimers can be used to efficiently target and deliver pesticides, herbicides, fertilizers or plant growth regulators in plants. Moreover, nanoscale carriers can anchor soil structure and soil organic matter to plant roots [157]. This process can slow down the uptake rate of active ingredients by plant roots, improve compounds 
stability, reduce the wastes produced, reduce their applied amount, and reduce costs $[157,158]$. Etxeberria et al. [159] used fluorescent NPs of different composition and sizes and followed their movement into citrus leaves by fluorescent microscopy. Their results indicate that in citrus leaves, the size exclusion limit for NPs is of $5.4 \mathrm{~nm}$. This conclusion was based on the capacity of PAMAM dendrimers G-4 and G-5 (4.5 and $5.4 \mathrm{~nm}$, respectively) to move through the cell wall and into the phloem, but the failure of similar PAMAM dendrimers G-6 $(6.7 \mathrm{~nm})$ to move through the apoplast. Dendrimer NPs with the size of $5.4 \mathrm{~nm}$ and smaller were observed to penetrate the leaf tissue, and then be taken up and mobilized by the phloem elements. This study provides evidence on the size limit for nanoparticle use in agriculture. The work of Santiago-Morales et al. [160] showed that amine-terminated third-generation (G3) PAMAM dendrimer affects normal seed germination of monocotyledonous and dicotyledonous species, such as Lolium perenne (ryegrass), Lycopersicon esculentum (tomato) and Lactuca sativa (lettuce). The toxicity of G3 PAMAM- $\left(\mathrm{NH}_{2}\right)_{32}$ for seed germination was low, with an $\mathrm{EC}_{10}$ in the order of $100 \mathrm{mg} \mathrm{L}^{-1}(14.5 \mu \mathrm{M})$. It is interesting to note that seeds exposed to a low concentration of dendrimer resulted in germination index (GI) above the control, this being particularly intense for Lactuca sativa.

Dendrimers find application as delivery agents to carry drugs, oligonucleotides or other molecules for agricultural utility as stated above. Dendrimers are important in increasing the solubility of active agents; improving the adhesion and penetration of active ingredients to plant surfaces; improving the water fastness of an active substance to the plant or seed; increasing the soil penetration of the active agents to reach the plant roots or under soil parts; and reducing the soil adhesion of the active ingredients to reach the plant roots. They can also provide protection against the enzymatic degradation of the active agent by plant or seed or microorganisms present in the soil [161].

The number of research data regarding the influencing effect of dendrimers on seed germination and seedling growth is scarce. Therefore, future research should focus on the dendrimer-triggered effects on plants in order to provide the theoretical basis of agricultural applications.

\subsection{The Effect of Composite Nanomaterials on Seed Germination}

Composite NMs or nanocomposites are hybrid materials produced by the combination of two or more materials with different properties.

Multiple mechanisms of action are associated with nanocomposites in biological systems, including the disruption of the cell wall and plasma membrane, the inhibition of protein synthesis and DNA replication, and an increase in the oxidation of cell components and compounds [162]. Different nanocomposites are also generally utilized by the industry as antimicrobial agents $[163,164]$, food packaging [165,166], the enhancement of plant physiological parameters [167] and against food oxidation [168]. Nanocomposites are basically divided into three different classes according to their matrix structures: ceramic matrix nanocomposites $\left(\mathrm{Al}_{2} \mathrm{O}_{3} / \mathrm{TiO}_{2}, \mathrm{Al}_{2} \mathrm{O}_{3} / \mathrm{SiO}_{2}, \mathrm{Al}_{2} \mathrm{O}_{3} / \mathrm{SiC}, \mathrm{Al}_{2} \mathrm{O}_{3} / \mathrm{CNT}\right)$; metal matrix nanocomposites $\left(\mathrm{Co} / \mathrm{Cr}, \mathrm{Fe}-\mathrm{Cr} / \mathrm{Al}_{2} \mathrm{O}_{3}, \mathrm{Fe}-\mathrm{MgO}\right)$; and polymer matrix nanocomposites (poliester/TiO 2 , polimer/CNT) $[162,169,170]$.

Chitosan, polylactic acid and hydroxyl-ethyl methacrylate-based NMs are examples of widely used nanocomposites. These NMs can be prepared easily and faster, with less raw material, unlike conventional polymers. Additionally, composite nanomaterials, which have high stability in biological fluids, have properties such as biodegradability, renewability and biocompatibility [171,172]. Singh et al. [173] studied the effect of nanoscale $\mathrm{TiO}_{2}$-activated carbon composite $\left(\mathrm{AC}-\mathrm{TiO}_{2}\right)$ on Solanum lycopersicum (L.) and Vigna radiata (L.) seed germination. Their results showed that the increase in nanocomposite concentration up to a certain level improves the germination rate and reduces the germination time. Accordingly, employing $\mathrm{AC}-\mathrm{TiO}_{2}$ nanocomposites at a suitable concentration may promote seed germination and also reduce the germination time in Solanum lycopersicum and Vigna radiata. As reported by Liu et al. [174], NMs could promote germination and rooting early for rice seeds and seedlings and the growth of rice at the tillering stage was obviously affected by nanocomposites. They indicated that the grain yield of rice and nitrogen agronomic utilization 
efficiency was increased after applying nano-carbon-incorporated SRF (slow release fertilizer). Abdel-Aziz et al. [175] studied how nano chitosan-NPK fertilizer enhanced the growth and productivity of Triticum aestivum (wheat) plants grown in sandy soil. Results indicated that all yield variables of wheat plants treated with increasing concentrations of nanocomposite NPK fertilizer (CS-PMAA-NPK (chitosan polymethacrylic acid nanoparticles loaded with nitrogen, phosphorus and potassium)), led to a significant increase in all growth parameters (root length, shoot length, fresh weight, dry weight, water content and leaf area), determined throughout the adult and reproductive growth and developmental stages.

The ability to develop these properties enables the use of composite materials in a variety of industries, including agriculture, energy, cosmetics and pharmaceuticals [176]. Today, applications of nanocomposites do exist, but the full potential is still not discovered [162]. Therefore, more research is needed to explore the effects of composite nanomaterials on seed germination and plant growth.

\section{Conclusions and Future Perspectives}

In this review, we attempted to emphasize that the increasing worldwide usage of NMs not only provides us technical, medical, industrial, agricultural, cosmetic novelties and conveniences but also creates challenges and possible hazards for all living organisms (including plants).

Due to excellent reports, we may presume that most of the NMs can enter the plants through the root epidermis, even at the germinative phase, which might be biotransformed into inonic form and translocated to the upper plant parts depending on several characters of the applied NMs such as the size, shape, stability, charge or coating. Since seed germination is the very early and sensitive phase of plant ontogenesis, it is important to determine how different NMs can influence it, especially in the case of crops.

From the reports overviewed, we may hypothesize that the stimulative effects of various NMs on seed germination are materialized via breaking seed dormancy, increasing germination rate and seedling vigor at the individual level, while at the cellular level, the upregulation of genes involved in cell division, increased antioxidant capacity or reprogrammed carbon/nitrogen metabolism is demonstrated.

The negative effects of different NMs in growing seedlings, according to the published results, are probably due to inter alia chromosomal aberrations and mitotic abnormalities (e.g., MWCNTs themselves are capable of binding to DNA), and therefore, reduced cell division in the root meristem and root shortening, hormonal imbalance, ROS/RNS overproduction and an increased level of lipid peroxidation, as summarized in Figure 4.

In agriculture, some kind of seed pre-treatment (i.e., seed priming) seems to be beneficial regarding seed germination efficiency, seedling growth or protection against pathogens, as it has been supported by many experimental data. We may suppose that nanopriming due to the presence of the seed coat generally provides a slow uptake of NMs by the developing plant, which upregulates the antioxidant defense system, resulting in better germination efficiency and plant growth.

We have promising data from germination tests about the dual effects of NMs on developing young plants, but we must take into account that plants usually grow in the soil, exposed to numerous internal and external factors, and thus, further realistic (open air, less controlled) experiments are needed to be carried out in addition to the laboratory studies, 


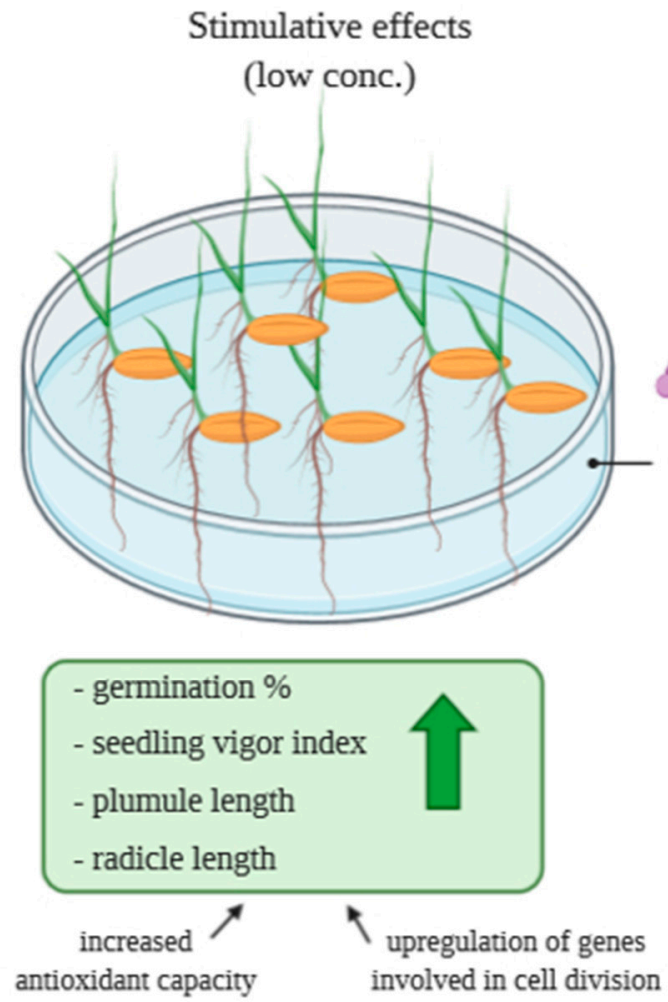

\section{Phytotoxic effects \\ (high conc.)}

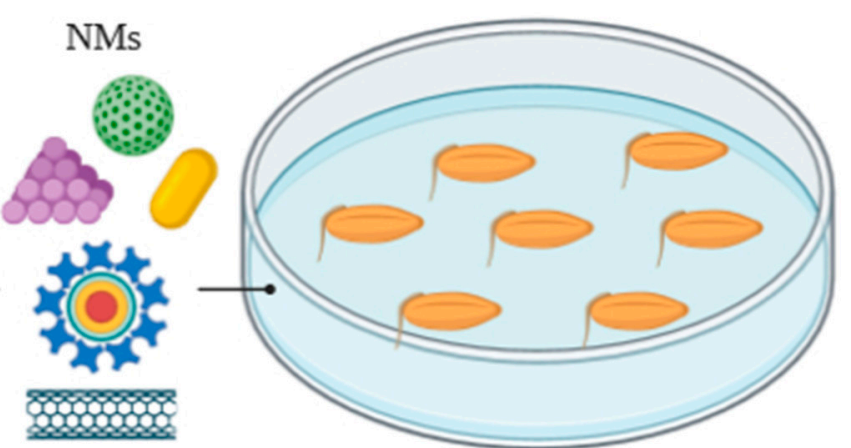

Figure 4. Schematic model summarizing the stimulative or inhibitory pathways of NMs on seed germination (figure created in BioRender.com).

Author Contributions: Conceptualization, R.S. and Z.K.; writing-original draft preparation, Z.K., Á.M., S.K. and R.S.; writing-review and editing, R.S.; visualization, Z.K., Á.M. and R.S.; supervision, R.S. All authors have read and agreed to the published version of the manuscript.

Funding: This work was supported by National Research, Development and Innovation Fund (Grant no. NKFI-1, KH129511 and K135303).

Conflicts of Interest: The authors declare no conflict of interest.

\section{References}

1. Schneider, J.J. Nanomaterials: Synthesis, properties and applications. In Advanced Materials; Edelstein, A.S., Cammarata, R.C., Eds.; IOP Publishing: Bristol, UK, 1996; p. 997.

2. British Standards Institution. Terminology for Nanomaterials; BSI: London, UK, 2007.

3. American Society for Testing and Materials. Standard Terminology Relating to Nanotechnology; E 2456-06; ASTM International: West Conshohocken, PA, USA, 2006.

4. Scientific Committee on Emerging and Newly Identified Health Risks. The Appropriateness of the Risk Assessment Methodology in Accordance with the Technical Guidance Documents for New and Existing Substances for Assessing the Risks of Nanomaterials; European Commission: Brussels, Belgium, 2007.

5. Piccinno, F.; Gottschalk, F.; Seeger, S.; Nowack, B. Industrial production quantities and uses of ten engineered nanomaterials in Europe and the world. J. Nano Res. 2012, 14, 1109-1112. [CrossRef]

6. Brar, S.K.; Verma, M.; Tyagi, R.D.; Surampalli, R.Y. Engineered nanoparticles in wastewater and wastewater sludge-Evidence and impacts. Waste Manag. 2010, 30, 504-520. [CrossRef] [PubMed]

7. Mageswari, A.; Srinivasan, R.; Subramanian, P.; Ramesh, N.; Gothandam, K.M. Nanomaterials: Classification, Biological Synthesis and Characterization. In Nanoscience in Food and Agriculture 3. Sustainable Agriculture Reviews; Ranjan, S., Dasgupta, N., Lichtfouse, E., Eds.; Springer: Cham, Switzerland, 2016; Volume 23, pp. 31-71. [CrossRef] 
8. Mahmoudi, M.; Simchi, A.; Milani, A.S.; Stroeve, P. Cell toxicity of superparamagnetic iron oxide nanoparticles. J. Colloid Interface Sci. 2009, 336, 510-518. [CrossRef] [PubMed]

9. Peralta-Videa, J.R.; Zhao, L.; Lopez-Moreno, M.L.; de la Rosa, G.; Hong, J.; Gardea-Torresdey, J.L. Nanomaterials and the environment: A review for the biennium 2008-2010. J. Hazard. Mater. 2011, 186, 1-15. [CrossRef] [PubMed]

10. Siegel, R.W. Synthesis and properties of nanophase materials. Mater. Sci. Eng. A 1993, 168, 189-197. [CrossRef]

11. Morton, W.J. Method of Dispersing Fluids. U.S. Patent 705,691, 29 July 1902.

12. Thenmozhi, S.; Dharmaraj, N.; Kadirvelu, K.; Kim, H.Y. Electrospun nanofibers: New generation materials for advanced applications. Mater. Sci. Eng. B 2017, 217, 36-48. [CrossRef]

13. Ramakrishna, S.; Fujihara, K.; Teo, W.-E.; Yong, T.; Ma, Z.; Ramaseshan, R. Electrospun nanofibers: Solving global issues. Mater. Today 2006, 9, 40-50. [CrossRef]

14. Hosseini, H.; Shojaee-Aliabadi, S.; Hosseini, S.M.; Mirmoghtadaie, L. Chapter 11-Nanoantimicrobials in food industry. In Nanotechnology Applications in Food; Oprea, A.E., Grumezescu, A.M., Eds.; Academic Press Elsevier: London, UK, 2017; pp. 223-243. [CrossRef]

15. Acosta, E. Bioavailability of nanoparticles in nutrient and nutraceutical delivery. Curr. Opin. Colloid Interface Sci. 2009, 14, 3-15. [CrossRef]

16. Tadros, T.; Izquierdo, P.; Esquena, J.; Solans, C. Formation and stability of nano-emulsions. Adv. Colloid Interface Sci. 2004, 108, 303-318. [CrossRef]

17. McClements, D.J.; Rao, J. Food-grade nanoemulsions: Formulation, fabrication, properties, performance, biological fate, and potential toxicity. Crit. Rev. Food Sci. Nutr. 2011, 51, 285-330. [CrossRef]

18. Murr, L.E.; Esquivel, E.V.; Bang, J.J.; de La Rosa, G.; Gardea-Torresdey, J.L. Chemistry and nanoparticulate compositions of a 10,000 year-old ice core melt water. Water Res. 2004, 38, 4282-4296. [CrossRef]

19. Verma, H.C.; Upadhyay, C.; Tripathi, A.; Tripathi, R.P.; Bhandari, N. Thermal decomposition pattern and particle size estimation of iron minerals associated with the Cretaceous-Tertiary boundary at Gubbio. Meteorit. Planet. Sci. 2002, 37, 901-909. [CrossRef]

20. Handy, R.D.; Owen, R.; Valsami-Jones, E. The ecotoxicology of nanoparticles and nanomaterials: Current status, knowledge gaps, challenges, and future needs. Ecotoxicology 2008, 17, 315-325. [CrossRef] [PubMed]

21. Buzea, C.; Pacheco, M. Nanomaterial and Nanoparticle: Origin and Activity. In Nanoscience and Plant-Soil Systems; Ghorbanpour, M., Manika, K., Varma, A., Eds.; Soil Biology, 48; Springer International Publishing: Cham, Switzerland, 2017; pp. 71-112. [CrossRef]

22. Lin, D.; Xing, B. Root uptake and phytotoxicity of ZnO nanoparticles. Environ. Sci. Technol. 2008, 42, 5580-5585. [CrossRef] [PubMed]

23. Ghodake, G.; Seo, Y.D.; Lee, D.S. Hazardous phytotoxic nature of cobalt and zinc oxide nanoparticles assessed using Allium cepa. J. Hazard. Mater. 2011, 186, 952-955. [CrossRef] [PubMed]

24. Zhang, Y.; Chen, Y.; Westerhoff, P.; Hristovski, K.; Crittenden, J.C. Stability of commercial metal oxide nanoparticles in water. Water Res. 2008, 42, 2204-2212. [CrossRef]

25. Lin, D.; Xing, B. Phytotoxicity of nanoparticles: Inhibition of seed germination and root growth. Environ. Pollut. 2007, 150, 243-250. [CrossRef]

26. Rico, C.M.; Majumdar, S.; Duarte-Gardea, M.; Peralta-Videa, J.R.; Gardea-Torresdey, J.L. Interaction of nanoparticles with edible plants and their possible implications in the food chain. J. Agric. Food Chem. 2011, 59, 3485-3498. [CrossRef]

27. Du, W.; Tan, W.; Peralta-Videa, J.R.; Gardea-Torresdey, J.L.; Ji, R.; Yin, Y.; Guo, H. Interaction of metal oxide nanoparticles with higher terrestrial plants: Physiological and biochemical aspects. Plant Phys. Biochem. 2017, 110, 210-225. [CrossRef]

28. Rizwan, M.; Ali, S.; Qayyum, M.F.; Ok, Y.S.; Adrees, M.; Ibrahim, M.; Zia-ur-Rehman, M.; Farid, M.; Abbas, F. Effect of metal and metal oxide nanoparticles on growth and physiology of globally important food crops: A critical review. J. Haz. Mat. 2017, 322, 2-16. [CrossRef] [PubMed]

29. Ahmed, B.; Rizvi, A.; Zaidi, A.; Khan, M.S.; Musarrat, J. Understanding the phyto-interaction of heavy metal oxide bulk and nanoparticles: Evaluation of seed germination, growth, bioaccumulation, and metallothionein production. RSC Adv. 2019, 9, 4210-4225. [CrossRef]

30. López-Moreno, M.L.; de la Rosa, G.; Hernandez-Viezcas, J.A.; Castillo-Michel, H.; Botez, C.E.; Peralta-Videa, J.R.; Gardea-Torresdey, J.L. Evidence of the differential biotransformation and genotoxicity of $\mathrm{ZnO}$ and $\mathrm{CeO}_{2}$ nanoparticles on soybean (Glycine max) plants. Environ. Sci. Technol. 2010, 44, 7315-7320. [CrossRef] [PubMed] 
31. Lv, J.; Zhang, S.; Luo, L.; Zhang, J.; Yang, K.; Christied, P. Accumulation, speciation and uptake pathway of ZnO nanoparticles in maize. Environ. Sci. Nano 2015, 2, 68. [CrossRef]

32. Molnar, A.; Papp, M.; Kovacs, D.Z.; Belteky, P.; Olah, D.; Feigl, G.; Szollosi, R.; Razga, Z.; Ordog, A.; Erdei, L.; et al. Nitro-oxidative signalling induced by chemically synthetized zinc oxide nanoparticles (ZnO NPs) in Brassica species. Chemosphere 2020, 251, 126419. [CrossRef] [PubMed]

33. Serag, M.F.; Kaji, N.; Gaillard, C.; Okamoto, Y.; Terasaka, K.; Jabasini, M.; Tokeshi, M.; Mizukami, H.; Bianco, A.; Baba, Y. Trafficking and subcellular localization of multiwalled carbon nanotubes in plant cells. ACS Nano 2011, 5, 493-499. [CrossRef] [PubMed]

34. Serag, M.F.; Kaji, N.; Habuchi, S.; Bianco, A.; Baba, Y. Nanobiotechnology meets plant cell biology: Carbon nanotubes as organelle targeting nanocarriers. RSC Adv. 2013, 3, 4856-4862. [CrossRef]

35. Verma, S.K.; Das, A.K.; Gantait, S.; Kumar, V.; Gurel, E. Applications of carbon nanomaterials in the plant system: A perspective view on the pros and cons. Sci. Total Environ. 2019, 667, 485-499. [CrossRef]

36. Ahmad, R.; Pranaw, K.; Khare, S.K. Effect of nanomaterials and their possible implication on the plants. In Plant Biotechnology: Progress in Genomic Era; Khurana, S.M.P., Gaur, R.K., Eds.; Springer: Singapore, 2019; pp. 213-229. [CrossRef]

37. Science Direct. Available online: https://www.sciencedirect.com/ (accessed on 15 October 2020).

38. Miralles, P.; Church, T.L.; Harris, A.T. Toxicity, uptake, and translocation of engineered nanomaterials in vascular plants. Environ. Sci. Technol. 2012, 46, 9224-9239. [CrossRef]

39. López-Serrano, A.; Olivas, R.M.; Landaluze, J.S.; Cámara, C. Nanoparticles: A global vision. Characterization, separation, and quantification methods. Potential environmental and health impact. Anal. Methods 2014, 6, 38-56. [CrossRef]

40. Raliya, R.; Saharan, V.; Dimkpa, C.; Biswas, P. Nanofertilizer for precision and sustainable agriculture: Current state and future perspectives. J. Agric. Food Chem. 2018, 66, 6487-6503. [CrossRef]

41. Radchuk, V.; Borisjuk, L. Physical, metabolic and developmental functions of the seed coat. Front. Plant Sci. 2014, 5, 510. [CrossRef] [PubMed]

42. Obroucheva, N.V. Aquaporins in seeds. Seed Sci. Res. 2013, 23, 213-216. [CrossRef]

43. Molnár, Á.; Rónavári, A.; Bélteky, P.; Szőllősi, R.; Valyon, E.; Oláh, D.; Rázga, Z.; Ördög, A.; Kónya, Z.; Kolbert, Z. ZnO nanoparticles induce cell wall remodeling and modify ROS/RNS signalling in roots of Brassica seedlings. Ecotoxic. Environ. Safe 2020, 206, 111158. [CrossRef] [PubMed]

44. Younes, N.A.; Hassan, H.S.; Elkady, M.F.; Hamed, A.M.; Dawood, M.F. Impact of synthesized metal oxide nanomaterials on seedlings production of three Solanaceae crops. Heliyon 2020, 6, e03188. [CrossRef]

45. Kroto, H.W.; Heath, J.R.; O’Brien, S.C.; Curl, R.F.; Smalley, R.E. C60: Buckminsterfullerene. Nature 1985, 318, 162-163. [CrossRef]

46. Iijima, S. Helical microtubules of graphitic carbon. Nature 1991, 354, 56-58. [CrossRef]

47. Dai, H. Carbon nanotubes: Synthesis, integration, and properties. Acc. Chem. Res. 2002, 35, 1035-1044. [CrossRef]

48. Dresselhaus, M.S.; Dai, H. Carbon nanotubes: Continued innovations and challenges. MRS Bull. 2004, 29, 237-243. [CrossRef]

49. Golberg, D.; Costa, P.M.F.J.; Mitome, M.; Bando, Y. Nanotubes in a gradient electric field, as revealed by STM-TEM technique. Nano Res. 2008, 1, 166-175. [CrossRef]

50. Service, R.F. Superstrong nanotubes show they are smart, too. Science 1998, 281, 893-894. [CrossRef]

51. Stampoulis, D.; Sinha, S.K.; White, J.C. Assay-Dependent Phytotoxicity of Nanoparticles to Plants. Environ. Sci. Technol. 2015, 43, 9473-9479. [CrossRef] [PubMed]

52. Park, S.; Ahn, Y.-J. Multi-walled carbon nanotubes and silver nanoparticles differentially affect seed germination, chlorophyll content, and hydrogen peroxide accumulation in carrot (Daucus carota L.). Biocatal. Agric. Biotechnol. 2016, 8, 257-262. [CrossRef]

53. Khodakovskaya, M.; Dervishi, E.; Mahmood, M.; Xu, Y.; Li, Z.; Watanabe, F.; Biris, A.S. Carbon nanotubes are able to penetrate plant seed coat and dramatically affect seed germination and plant growth. ACS Nano 2009, 3, 3221-3227. [CrossRef] [PubMed]

54. Khodakovskaya, M.V.; de Silva, K.; Biris, A.S.; Dervishi, E.; Villagarcia, H. Carbon nanotubes induce growth enhancement of tobacco cells. ACS Nano 2012, 6, 2128-2135. [CrossRef] 
55. Cañas, J.E.; Long, M.; Nations, S.; Vadan, R.; Dai, L.; Luo, M.; Ambikapathi, R.; Lee, E.H.; Olszyk, D. Effects of functionalized and nonfunctionalized single-walled carbon nanotubes on root elongation of select crop species. Environ. Toxicol. Chem. 2009, 27, 1922-1931. [CrossRef] [PubMed]

56. Liu, Q.; Chen, B.; Wang, Q.; Shi, X.; Xiao, Z.; Lin, J.; Fang, X. Carbon Nanotubes as Molecular Transporters for Walled Plant Cells. Nano Lett. 2009, 9, 1007-1010. [CrossRef]

57. Vishwakarma, K.; Sharma, S.; Narayan, R.P.; Srivastava, P.; Khan, A.S.; Dubey, N.K.; Tripathi, D.K.; Chauhan, D.K. Plants and carbon nanotubes (CNTs) interface: Present status and future prospects. In Nanotechnology; Prasad, R., Kumar, V., Kumar, M., Eds.; Springer: Singapore, 2017; pp. 317-340. [CrossRef]

58. Srinivasan, C.; Ramiah, S. Nano-agriculture-Carbon nanotubes enhance tomato seed germination and plant growth. Curr. Sci. 2010, 99, 274-275.

59. Ghodake, G.; Seo, Y.D.; Park, D.; Lee, D.S. Phytotoxicity of Carbon Nanotubes Assessed by Brassica Juncea and Phaseolus Mungo. J. Nanoelectron. Optoelectron. 2010, 5, 157-160. [CrossRef]

60. Haghighi, M.; da Silva, J.A.T. The Effect of Carbon Nanotubes on the Seed Germination and Seedling Growth of Four Vegetable Species. J. Crop Sci. Biotech. 2014, 17, 201-208. [CrossRef]

61. Pourkhaloee, A.; Haghighi, M.; Saharkhiz, M.J.; Jouzi, H.; Doroodmand, M.M. Carbon Nanotubes Can Promote Seed Germination via Seed Coat Penetration. Seed Technol. 2011, 33, 155-169.

62. Srivastava, A.; Rao, D.P. Enhancement of Seed Germination and Plant Growth of Wheat, Maize, Peanut and Garlic Using Multiwalled Carbon Nanotubes. Eur. Chem. Bull. 2014, 3, 502-504.

63. Jiang, Y.; Hua, Z.; Zhao, Y.; Liu, Q.; Wang, F.; Zhang, Q. The Effect of Carbon Nanotubes on Rice Seed Germination and Root Growth. In Proceedings of the 2012 International Conference on Applied Biotechnology (ICAB 2012); Lecture Notes in Electrical Engineering; Zhang, T.C., Ouyang, P., Kaplan, S., Skarnes, B., Eds.; Springer: Berlin/Heidelberg, Germany, 2014; Volume 250. [CrossRef]

64. Lahiani, M.H.; Dervishi, E.; Chen, J.; Nima, Z.; Gaume, A.; Biris, A.S.; Khodakovskaya, M.V. Impact of Carbon Nanotube Exposure to Seeds of Valuable Crops. ACS Appl. Mater. Interfaces 2013, 5, 7965-7973. [CrossRef] [PubMed]

65. Lahiani, M.H.; Chen, J.; Irin, F.; Puretzky, A.A.; Green, M.J.; Khodakovskaya, M.V. Interaction of carbon nanohorns with plants: Uptake and biological effects. Carbon 2015, 81, 607-619. [CrossRef]

66. Tripathi, S.; Sonkar, S.K.; Sarkar, S. Growth stimulation of gram (Cicer arietinum) plant by water soluble carbon nanotubes. Nanoscale 2011, 3, 1176-1181. [CrossRef]

67. Mukherjee, A.; Majumdar, S.; Servin, A.D.; Pagano, L.; Dhankher, O.P.; White, J.C. Carbon Nanomaterials in Agriculture: A Critical Review. Front. Plant Sci. 2016, 7, 172. [CrossRef] [PubMed]

68. Vithanage, M.; Seneviratne, M.; Ahmad, M.; Sarkar, B.; Ok, Y.S. Contrasting effects of engineered carbon nanotubes on plants: A review. Environ. Geochem. Health 2017, 39, 1421-1439. [CrossRef]

69. Hao, Y.; Yu, F.; Lv, R.; Ma, C.; Zhang, Z.; Rui, Y.; Liu, L.; Cao, W.; Xing, B. Carbon Nanotubes Filled with Different Ferromagnetic Alloys Affect the Growth and Development of Rice Seedlings by Changing the C:N Ratio and Plant Hormones Concentrations. PLoS ONE 2016, 11, e0157264. [CrossRef]

70. Hatami, M.; Hadian, J.; Ghorbanpour, M. Mechanisms underlying toxicity and stimulatory role of single-walled carbon nanotubes in Hyoscyamus niger during drought stress simulated by polyethylene glycol. J. Hazard. Mater. 2017, 324, 306-320. [CrossRef]

71. Khalifa, N.S. The Effect of Multi-walled Carbon Nanotubes on Pennycress (Thlaspi arvense L.). Plant 2018, 58, 529-537. [CrossRef]

72. Pandey, K.; Lahiani, M.H.; Hicks, V.K.; Hudson, M.K.; Green, M.J.; Khodakovskaya, M. Effects of carbon-based nanomaterials on seed germination, biomass accumulation and salt stress response of bioenergy crops. PLoS ONE 2018, 13, e0202274. [CrossRef]

73. González-García, Y.; López-Vargas, E.R.; Cadenas-Pliego, G.; Benavides-Mendoza, A.; González-Morales, S.; Robledo-Olivo, A.; Alpuche-Solís, Á.G.; Juárez-Maldonado, A. Impact of Carbon Nanomaterials on the Antioxidant System of Tomato Seedlings. Int. J. Mol. Sci. 2019, 20, 5858. [CrossRef] [PubMed]

74. Ali, M.H.; Sobze, J.-M.; Pham, T.H.; Nadeem, M.; Liu, C.; Galagedara, L.; Cheema, M.; Thomas, R. Carbon Nanoparticles Functionalized with Carboxylic Acid Improved the Germination and Seedling Vigor in Upland Boreal Forest Species. Nanomaterials 2020, 10, 176. [CrossRef] [PubMed] 
75. López-Vargas, E.R.; González-García, Y.; Pérez-Álvarez, M.; Cadenas-Pliego, G.; González-Morales, S.; Benavides-Mendoza, A.; Cabrera, R.I.; Juárez-Maldonado, A. Seed Priming with Carbon Nanomaterials to Modify the Germination, Growth, and Antioxidant Status of Tomato Seedlings. Agronomy 2020, 10, 639. [CrossRef]

76. Chang, X.; Song, Z.; Xu, Y.; Gao, M. Effects of carbon nanotubes on growth of wheat seedlings and Cd uptake. Chemosphere 2020, 240, 124931. [CrossRef]

77. Wenli, S.; Shahrajabian, M.H.; Huang, Q. Soybean Seeds Treated with Single Walled Carbon Nanotubes (SwCNTs) Showed Enhanced Drought Tolerance During Germination. Int. J. Adv. Biol. Biomed. Res. 2020, 8, 9-16.

78. Baz, H.; Creech, M.; Chen, J.; Gong, H.; Bradford, K.; Huo, H. Water-Soluble Carbon Nanoparticles Improve Seed Germination and Post-Germination Growth of Lettuce under Salinity Stress. Agronomy 2020, 10, 1192. [CrossRef]

79. Chen, J.; Dou, R.; Yang, Z.; Wang, X.; Mao, C.; Gao, X.; Wang, L. The effect and fate of water-soluble carbon nanodots in maize (Zea mays L.). Nanotoxicology 2016, 10, 818-828. [CrossRef]

80. Wang, H.; Zhang, M.; Song, Y.; Li, H.; Huang, H.; Shao, M.; Liu, Y.; Kang, Z. Carbon dots promote the growth and photosynthesis of mung bean sprouts. Carbon 2018, 136, 94-102. [CrossRef]

81. Wang, A.; Kang, F.; Wang, Z.; Shao, Q.; Li, Z.; Zhu, G.; Lu, J.; Li, Y.Y. Facile Synthesis of Nitrogen-Rich Carbon Dots as Fertilizers for Mung Bean Sprouts. Adv. Sustain. Syst. 2019, 3, 1800132. [CrossRef]

82. Qian, K.; Guo, H.; Chen, G.; Ma, C.; Xing, B. Distribution of different surface modified carbon dots in pumpkin seedlings. Sci. Rep. 2018, 8. [CrossRef]

83. Li, Y.; Xu, X.; Wu, Y.; Zhuang, J.; Zhang, X.; Zhang, H.; Lei, B.; Hu, C.; Liu, Y. A review on the effects of carbon dots in plant systems. Mater. Chem. Front. 2020, 4, 437-448. [CrossRef]

84. Chen, Q.; Chen, L.; Nie, X.; Man, H.; Guo, Z.; Wang, X.; Tu, J.; Jin, G.; Ci, L. Impacts of surface chemistry of functional carbon nanodots on the plant growth. Ecotox. Environ. Safe 2020, 206, 111220. [CrossRef] [PubMed]

85. Sun, L.; Wang, R.; Ju, Q.; Xu, J. Physiological, Metabolic, and Transcriptomic Analyses Reveal the Responses of Arabidopsis Seedlings to Carbon Nanohorns. Environ. Sci. Technol. 2020, 54, 4409-4420. [CrossRef] [PubMed]

86. Liu, Q.; Zhao, Y.; Wan, Y.; Zheng, J.; Zhang, X.; Wang, C.; Fang, X.; Lin, J. Study of the inhibitory effect of water-soluble fullerenes on plant growth at the cellular level. ACS Nano 2010, 4, 5743-5748. [CrossRef] [PubMed]

87. Gao, J.; Wang, Y.; Folta, K.M.; Krishna, V.; Bai, W.; Indeglia, P.; Georgieva, A.; Nakamura, H.; Koopman, B.; Moudgil, B. Polyhydroxy Fullerenes (Fullerols or Fullerenols): Beneficial Effects on Growth and Lifespan in Diverse Biological Models. PLoS ONE 2011, 6, e19976. [CrossRef]

88. Panova, G.; Ktitorova, I.; Skobeleva, O.; Sinjavina, N.; Charykov, N.; Semenov, K. Impact of polyhydroxy fullerene (fullerol or fullerol) on growth and biophysical characteristics of barley seedlings in favourable and stressful conditions. Plant Growth Regul. 2016, 79, 309-317. [CrossRef]

89. Xiong, J.-L.; Li, J.; Wang, H.-C.; Zhang, C.-L.; Naeem, M.S. Fullerol improves seed germination, biomass accumulation, photosynthesis and antioxidant system in Brassica napus L. under water stress. Plant Physiol. Biochem. 2018, 129, 130-140. [CrossRef]

90. Liu, Y.; Wang, T.; Cao, J.; Zang, Z.; Wu, Q.; Wang, H.; Tai, F.; He, R. Quaternary Ammonium Salts of Iminofullerenes: Fabrication and Effect on Seed Germination. J. Agric. Food Chem. 2019, 67, 13509-13517. [CrossRef]

91. Nair, R.; Mohamed, M.S.; Gao, W.; Maekawa, T.; Yoshida, Y.; Ajayan, P.M.; Kumar, D.S. Effect of carbon nanomaterials on the germination and growth of rice plants. J. Nanosci. Nanotechnol. 2012, 12, 2212-2220. [CrossRef]

92. Zhang, M.; Gao, B.; Chen, J.; Li, Y. Effects of graphene on seed germination and seedling growth. J. Nanopart. Res. 2015, 17, 78. [CrossRef]

93. Liu, S.; Wei, H.; Li, Z.; Li, S.; Yan, H.; He, Y.; Tian, Z. Effects of graphene on germination and seedling morphology in rice. J. Nanosci. Nanotechnol. 2015, 15, 2695-2701. [CrossRef] [PubMed]

94. Zhang, P.; Zhang, R.; Fang, X.; Song, T.; Cai, X.; Liu, H.; Du, S. Toxic effects of graphene on the growth and nutritional levels of wheat (Triticum aestivum L.): Short-and longterm exposure studies. J. Hazard. Mater. 2016, 317, 543-551. [CrossRef] [PubMed]

95. Ren, W.; Chang, H.; Teng, Y. Sulfonated graphene-induced hormesis is mediated through oxidative stress in the roots of maize seedlings. Sci. Total Environ. 2016, 572, 926-934. [CrossRef] 
96. Chen, J.; Yang, L.; Li, S.; Ding, W. Various Physiological Response to Graphene Oxide and Amine-Functionalized Graphene Oxide in Wheat (Triticum aestivum). Molecules 2018, 23, 1104. [CrossRef] [PubMed]

97. Vochita, G.; Opric, L.; Gherghel, D.; Mihai, C.-T.; Boukherrou, R.; Lobiu, A. Graphene oxide effects in early ontogenetic stages of Triticum aestivum L. seedlings. Ecotoxic. Environ. Safe 2019, 181, 345-352. [CrossRef] [PubMed]

98. Xu, Y.; Lu, Y.; Li, J.; Liu, R.; Zhu, X. Effect of graphene quantum dot size on plant growth. Nanoscale 2020, 12, 15045-15049. [CrossRef] [PubMed]

99. Koo, Y.; Wang, J.; Zhang, Q.; Zhu, H.; Chehab, E.W.; Colvin, V.L.; Alvarez, P.J.; Braam, J. Fluorescence reports intact quantum dot uptake into roots and translocation to leaves of Arabidopsis thaliana and subsequent ingestion by insect herbivores. Environ. Sci. Technol. 2015, 49, 626-632. [CrossRef]

100. Li, X.Q.; Elliott, D.W.; Zhang, W.X. Zero-valent iron nanoparticles for abatement of environmental pollutants: Materials and engineering aspects. Crit. Rev. Solid State Mater. Sci. 2006, 31, 111-122. [CrossRef]

101. Zhang, W. Nanoscale iron particles for environmental remediation: An overview. J. Nanopart. Res. 2003, 5, 323-332. [CrossRef]

102. Klopffer, W.; Curran, M.A.; Frankl, P.; Heijungs, R.; Kohler, A.; Olsen, S.I. Nanotechnology and life cycle assessment. In A Systems Approach to Nanotechnology and the Environment: Synthesis of Results Obtained at a Workshop; The European Commission and Woodrow Wilson International Center for Scholars: Washington, DC, USA, 2006.

103. Kramer, J.R.; Benoit, G.; Bowles, K.C.; Di Toro, D.M.; Herrin, R.T.; Luther, G.W.; Manalopoulis, H.; Robilliard, K.A.; Shafer, M.M.; Shaw, J.R. Environmental chemistry of silver. In Silver in the Environment: Transport, Fate, and Effects; Andren, A.W., Bober, T.W., Eds.; SETAC: Pensacola, FL, USA, 2002; pp. 1-25.

104. Doty, R.C.; Tshikhudo, T.R.; Brust, M. Extremely stablewatersoluble Ag nanoparticles. Chem. Mater. 2005, 17, 4630-4635. [CrossRef]

105. Barrena, R.; Casals, E.; Colón, J.; Font, X.; Sánchez, A.; Puntes, V. Evaluation of the ecotoxicity of model nanoparticles. Chemosphere 2009, 75, 850-857. [CrossRef] [PubMed]

106. Parveen, A.; Mazhari, B.B.Z.; Rao, S. Impact of bio-nanogold on seed germination and seedling growth in Pennisetum glaucum. Enzyme Microb. Technol. 2016, 95, 107-111. [CrossRef] [PubMed]

107. Almutairi, Z.M.; Alharbi, A. Effect of silver nanoparticles on seed germination of crop plants. J. Adv. Agric. 2015, 4, 283-288. [CrossRef]

108. Gorka, D.E.; Liu, J. Effect of direct contact on the phytotoxicity of silver nanomaterials. Environ. Sci. Technol. 2016, 50, 10370-10376. [CrossRef] [PubMed]

109. Prażak, R.; Święciło, A.; Krzepiłko, A.; Michałek, S.; Arczewska, M. Impact of Ag Nanoparticles on Seed Germination and Seedling Growth of Green Beans in Normal and Chill Temperatures. Agriculture 2020, 10, 312. [CrossRef]

110. Bouguerra, S.; Gavina, A.; da Graça Rasteiro, M.; Rocha-Santos, T.; Ksibi, M.; Pereira, R. Effects of cobalt oxide nanomaterial on plants and soil invertebrates at different levels of biological organization. J. Soils Sediments 2019, 19, 3018-3034. [CrossRef]

111. Hatami, M.; Ghorbanpour, M.; Salehiarjomand, H. Nano-anatase $\mathrm{TiO}_{2}$ modulates the germination behavior and seedling vigority of some commercially important medicinal and aromatic plants. J. Environ. Biol. 2014, 8, 53-59.

112. Prasad, T.N.V.K.V.; Sudhakar, P.; Sreenivasulu, Y.; Latha, P.; Munaswamy, V.; Reddy, K.R.; Sreeprasad, T.S.; Sajanlal, P.R.; Pradeep, T. Effect of nanoscale zinc oxide particles on the germination, growth and yield of peanut. J. Plant. Nutr. 2012, 35, 905-927. [CrossRef]

113. Afrayeem, S.M.; Chaurasia, A.K. Effect of zinc oxide nanoparticles on seed germination and seed vigour in chilli (Capsicum annuum L.). J. Pharmacogn. Phytochem. 2017, 6, 1564-1566.

114. García-López, I.J.; Lira-Saldivar, R.H.; Zavala-García, F.; Olivares-Sáenz, E.; Niño-Medina, G.; Angélica Ruiz-Torres, N.; Méndez-Argüello, B.; Díaz-Barriga, E. Effects of zinc oxide nanoparticles on growth and antioxidant enzymes of Capsicum chinense. Toxicol. Environ. Chem. 2018, 100, 560-572. [CrossRef]

115. de la Rosa, G.; López-Moreno, M.L.; de Haro, D.; Botez, C.E.; Peralta-Videa, J.R.; Gardea-Torresdey, J.L. Effects of $\mathrm{ZnO}$ nanoparticles in alfalfa, tomato, and cucumber at the germination stage: Root development and X-ray absorption spectroscopy studies. Pure Appl. Chem. 2013, 85, 2161-2174. [CrossRef] 
116. Jayarambabu, N.; Kumari, B.S.; Rao, K.V.; Prabhu, Y.T. Germination and growth characteristics of mungbean seeds (Vigna radiata L.) affected by synthesized zinc oxide nanoparticles. Int. J. Curr. Eng. Technol. 2014, 4, 2347-5161.

117. Srinivasan, R.; Maity, A.; Singh, K.K.; Ghosh, P.K.; Kumar, S.; Srivastava, M.K.; Radhakrishna, A.; Srivastava, R.; Kumari, B. Influence of copper oxide and zinc oxide nano-particles on growth of fodder cowpea and soil microbiological properties. Range Manag. Agrofor. 2017, 38, 208-214.

118. Bayramzadeh, V.; Ghadiri, M.; Davoodi, M.H. Effects of silver nanoparticle exposure on germination and early growth of Pinus sylvestris and Alnus subcordata. Sains Malays. 2019, 48, 937-944. [CrossRef]

119. Parveen, A.; Rao, S. Effect of nanosilver on seed germination and seedling growth in Pennisetum glaucum. J. Clust. Sci. 2015, 26, 693-701. [CrossRef]

120. Razzaq, A.; Ammara, R.; Jhanzab, H.M.; Mahmood, T.; Hafeez, A.; Hussain, S. A novel nanomaterial to enhance growth and yield of wheat. J. Nanosci. Technol. 2016, 2, 55-58.

121. López-Moreno, M.L.; de la Rosa, G.; Hernández-Viezcas, J.A.; Peralta-Videa, J.R.; Gardea-Torresdey, J.L. X-ray absorption spectroscopy (XAS) corroboration of the uptake and storage of $\mathrm{CeO}_{2}$ nanoparticles and assessment of their differential toxicity in four edible plant species. J. Agric. Food Chem. 2010, 58, 3689-3693. [CrossRef]

122. Naskar, A.; Goswami, M.; Ghosh, A.G.R. Effects of Iron Oxide Nanoparticles on Chick Pea (Cicer arietinum): Physiological Profiling, Chlorophylls Assay and Antioxidant Potential. Int. Res. J. Eng. Technol. 2020, 7, 3001-3003.

123. Hao, Y.; Zhang, Z.T.; Rui, Y.K.; Ren, J.Y.; Hou, T.Q.; Wu, S.J.; Rui, M.M.; Jiang, F.P.; Liu, L.M. Effect of different nanoparticles on seed germination and seedling growth in rice. In Proceedings of the 2nd Annual International Conference on Advanced Material Engineering, AME 2016, Wuhan, China, 15-17 April 2016; Atlantis Press: Paris, France, 2016; pp. 166-173.

124. Mahmoodzadeh, H.; Nabavi, M.; Kashefi, H. Effect of nanoscale titanium dioxide particles on the germination and growth of canola (Brassica napus). J. Ornam. Hortic. 2013, 3, 25-32.

125. Laware, S.L.; Raskar, S. Effect of titanium dioxide nanoparticles on hydrolytic and antioxidant enzymes during seed germination in onion. Int. J. Curr. Microbiol. App. Sci. 2014, 3, 749-760.

126. Laware, S.L.; Raskar, S. Influence of Zinc Oxide nanoparticles on growth, flowering and seed productivity in onion. Int. J. Curr. Microbiol. App. Sci. 2014, 3, 874-881.

127. Maity, A.; Natarajan, N.; Vijay, D.; Srinivasan, R.; Pastor, M.; Malaviya, D.R. Influence of Metal Nanoparticles (NPs) on Germination and Yield of Oat (Avena sativa) and Berseem (Trifolium alexandrinum). Proc. Natl. Acad. Sci. USA India Sect. B Biol. Sci. 2018, 88, 595-607. [CrossRef]

128. Doğaroğlu, Z.G.; Köleli, N. $\mathrm{TiO}_{2}$ and $\mathrm{ZnO}$ nanoparticles toxicity in barley (Hordeum vulgare L.). Clean-Soil Air Water 2017, 45, 1700096. [CrossRef]

129. Boonyanitipong, P.; Kositsup, B.; Kumar, P.; Baruah, S.; Dutta, J. Toxicity of ZnO and $\mathrm{TiO}_{2}$ nanoparticles on germinating rice seed Oryza sativa L. Int. J. Biosci. Biochem. Bioinformat. 2011, 1, 282-285. [CrossRef]

130. Kumar, S.; Patra, A.K.; Datta, S.C.; Rosin, K.G.; Purakayastha, T.J. Phytotoxicity of nanoparticles to seed germination of plants. Int. J. Adv. Res. 2015, 3, 854-865.

131. Jain, N.; Bhargava, A.; Pareek, V.; Akhtar, M.S.; Panwar, J. Does seed size and surface anatomy play role in combating phytotoxicity of nanoparticles? Ecotoxicology 2017, 26, 238-249. [CrossRef] [PubMed]

132. Medina-Velo, I.A.; Barrios, A.C.; Zuverza-Mena, N.; Hernandez-Viezcas, J.A.; Chang, C.H.; Ji, Z.; Zink, J.I.; Peralta-Videa, J.R.; Gardea-Torresdey, J.L. Comparison of the effects of commercial coated and uncoated $\mathrm{ZnO}$ nanomaterials and Zn compounds in kidney bean (Phaseolus vulgaris) plants. J. Hazard. Mat. 2017, 332, 214-222. [CrossRef] [PubMed]

133. Raliya, R.; Nair, R.; Chavalmane, S.; Wang, W.N.; Biswas, P. Mechanistic evaluation of translocation and physiological impact of titanium dioxide and zinc oxide nanoparticles on the tomato (Solanum lycopersicum L.) plant. Metallomics 2015, 7, 1584-1594. [CrossRef]

134. Geisler-Lee, J.; Wang, Q.; Yao, Y.; Zhang, W.; Geisler, M.; Li, K.; Huang, Y.; Chen, Y.; Kolmakov, A.; Ma, X. Phytotoxicity, accumulation and transport of silver nanoparticles by Arabidopsis thaliana. Nanotoxicology 2012, 7, 323-337. [CrossRef]

135. Lee, W.M.; An, Y.J.; Yoon, H.; Kweon, H.S. Toxicity and bioavailability of copper nanoparticles to the terrestrial plants mung bean (Phaseolus radiatus) and wheat (Triticum aestivum): Plant agar test for water-insoluble nanoparticles. Environ. Toxicol. Chem. 2008, 27, 1915-1921. [CrossRef] 
136. Yang, Z.; Chen, J.; Dou, R.; Gao, X.; Mao, C.; Wang, L. Assessment of the phytotoxicity of metal oxide nanoparticles on two crop plants, maize (Zea mays L.) and rice (Oryza sativa L.). Int. J. Environ. Res. Public Health 2015, 12, 15100-15109. [CrossRef]

137. Lee, C.W.; Mahendra, S.; Zodrow, K.; Li, D.; Tsai, Y.C.; Braam, J.; Alvarez, P.J. Developmental phytotoxicity of metal oxide nanoparticles to Arabidopsis thaliana. Environ. Toxicol. Chem. 2010, 29, 669-675. [CrossRef] [PubMed]

138. Castiglione, M.R.; Giorgetti, L.; Geri, C.; Cremonini, R. The effects of nano-TiO 2 on seed germination, development and mitosis of root tip cells of Vicia narbonensis L. and Zea mays L. J. Nanopart. Res. 2011, 13, 2443-2449. [CrossRef]

139. Mousavi Kouhi, S.M.; Lahouti, M.; Ganjeali, A.; Entezari, M.H. Comparative phytotoxicity of ZnO nanoparticles, $\mathrm{ZnO}$ microparticles, and $\mathrm{Zn}^{2+}$ on rapeseed (Brassica napus L.): Investigating a wide range of concentrations. Toxicol. Environ. Chem. 2014, 96, 861-868. [CrossRef]

140. Hafeez, A.; Razzaq, A.; Mahmood, T.; Jhanzab, H.M. Potential of copper nanoparticles to increase growth and yield of wheat. J. Nanosci. Adv. Technol. 2015, 1, 6-11.

141. Zuverza-Mena, N.; Medina-Velo, I.A.; Barrios, A.C.; Tan, W.; Peralta-Videa, J.R.; Gardea-Torresdey, J.L. Copper nanoparticles/compounds impact agronomic and physiological parameters in cilantro (Coriandrum sativum). Environ. Sci. Process. Impacts 2015, 17, 783-1793. [CrossRef]

142. Mirzaei, H.; Darroudi, M. Zinc oxide nanoparticles: Biological synthesis and biomedical applications. Ceram. Int. 2017, 43, 907-914. [CrossRef]

143. Balážová, L'.; Babula, P.; Baláž, M.; Bačkorová, M.; Bujňáková, Z.; Briančin, J.; Kurmanbayeva, A.; Sagi, M. Zinc oxide nanoparticles phytotoxicity on halophyte from genus Salicornia. Plant Phys. Biochem. 2018, 130, 30-42. [CrossRef]

144. Nair, P.M.G.; Chung, I.M. Regulation of morphological, molecular and nutrient status in Arabidopsis thaliana seedlings in response to ZnO nanoparticles and Zn ion exposure. Sci. Total Environ. 2017, 575, 187-198. [CrossRef]

145. Potters, G.; Pasternak, T.P.; Guisez, Y.; Palme, K.J.; Jansen, M.A. Stress-induced morphogenic responses: Growing out of trouble? Trends Plant. Sci. 2007, 12, 98-105. [CrossRef]

146. Adhikari, T.; Kundu, S.; Biswas, A.K.; Tarafdar, J.C.; Rao, A.S. Effect of copper oxide nano particle on seed germination of selected crops. J. Agric. Sci. Technol. 2012, 2, 815-823.

147. Rout, G.R.; Sahoo, S. Role of iron in plant growth and metabolism. Agric. Rev. 2015, 3, 1-24. [CrossRef]

148. Alivisatos, A.P.; Gu, W.; Larabell, C. Quantum dots as cellular probes. Annu. Rev. Biomed. Eng. 2005, 7, 55-76. [CrossRef]

149. Nair, R.; Poulose, A.C.; Nagaoka, Y.; Yoshida, Y.; Maekawa, T.; Kumar, D.S. Uptake of FITC labeled silica nanoparticles and quantum dots by rice seedlings: Effects on seed germination and their potential as biolabels for plants. J. Fluoresc. 2011, 21, 2057. [CrossRef]

150. Das, S.; Wolfson, B.P.; Tetard, L.; Tharkur, J.; Bazata, J.; Santra, S. Effect of N-acetyl cysteine coated CdS: $\mathrm{Mn} / \mathrm{ZnS}$ quantum dots on seed germination and seedling growth of snow pea (Pisum sativum L.): Imaging and spectroscopic studies. Environ. Sci. Nano 2015, 2, 203-212. [CrossRef]

151. Svenson, S.; Tomalia, D.A. Dendrimers in biomedical applications-Reflections on the field. Adv. Drug Deliv. Rev. 2005, 57, 2106-2129. [CrossRef]

152. Lee, C.C.; MacKay, J.A.; Fre'chet, J.M.; Szoka, F.C. Designing dendrimers for biological applications. Nat. Biotechnol. 2005, 23, 1517-1526. [CrossRef] [PubMed]

153. Roveda Júnior, A.C.; Franco, D.W. Nitric oxide releasing-dendrimers: An overview. Braz. J. Pharm. Sci. 2013, 49, 1-14. [CrossRef]

154. Seabra, A.B.; Justo, G.Z.; Haddad, P.S. State of the art, challenges and perspectives in the design of nitric oxide-releasing polymeric nanomaterials for biomedical applications. Biotechnol. Adv. 2015, 33, 1370-1379. [CrossRef]

155. Klaine, S.J.; Alvarez, P.J.; Batley, G.E.; Fernandes, T.F.; Handy, R.D.; Lyon, D.Y.; Mahendra, S.; McLaughlin, M.J.; Lead, J.R. Nanomaterials in the environment: Behavior, fate, bioavailability, and effects. Environ. Toxicol. Chem. 2008, 27, 1825-1851. [CrossRef]

156. NAAS. Nanotechnology in Agri-Food: Scope and Current Relevance; Policy Paper No. 63; National Academy of Agricultural Sciences: New Delhi, India, 2013; p. 20.

157. Ditta, A. How helpful is nanotechnology in agriculture? Adv. Nat. Sci. Nanosci. Nanotechnol. 2012, 3, 3-5. [CrossRef] 
158. Dhewa, T. Nanotechnology applications in agri-food: An update. Octa J. Environ. Res. 2015, 3, $204-211$.

159. Etxeberria, E.; Gonzalez, P.; Bhattacharya, P.; Sharma, P.; Ke, P.C. Determining the size exclusion for nanoparticles in Citrus leaves. HortScience 2016, 51, 732-737. [CrossRef]

160. Santiago-Morales, J.; Rosal, R.; Hernando, M.D.; Ulaszewska, M.M.; Calvo, E.G.; Alba, A.R.F. Fate and transformation products of amine-terminated PAMAM dendrimers under ozonation and irradiation. J. Haz. Mat. 2014, 266, 102-113. [CrossRef]

161. Kaphle, A.; Navya, P.N.; Umapathi, A.; Daima, H.K. Nanomaterials for agriculture, food and environment: Applications, toxicity and regulation. Environ. Chem. Lett. 2018, 16, 43-58. [CrossRef]

162. Omanović-Mikličanin, E.; Badnjević, A.; Kazlagić, A.; Hajlovac, M. Nanocomposites: A brief review. Health Technol. 2020, 10, 51-59. [CrossRef]

163. Kawahara, K.; Tsuruda, K.; Morishita, M.; Uchida, M. Antibacterial effect of silver-zeolite on oral bacteria under anaerobic conditions. Dent. Mater. 2000, 16, 452-455. [CrossRef]

164. Matsumura, Y.; Yoshikata, K.; Kunisaki, S.; Tsuchido, T. Mode of bactericidal action of silver zeolite and its comparison with that of silver nitrate. Appl. Environ. Microbiol. 2003, 69, 4278-4281. [CrossRef]

165. Arora, A.; Padua, G.W. Review: Nanocomposites in food packaging. J. Food Sci. 2010, 75, R43-R49. [CrossRef]

166. Chaudhry, Q.; Scotter, M.; Blackburn, J.; Ross, B.; Boxall, A.; Castle, L.; Aitken, R.; Watkins, R. Applications and implications of nanotechnologies for the food sector. Food Addit. Contam. Part A Chem. Anal. Control Expo. Risk Assess. 2008, 25, 241-258. [CrossRef]

167. Kole, C.; Kole, P.; Randunu, K.M.; Choudhary, P.; Podila, R.; Ke, P.C.; Rao, A.M.; Marcus, R.K. Nanobiotechnology can boost crop production and quality: First evidence from increased plant biomass, fruit yield and phytomedicine content in bitter melon (Momordica charantia). BMC Biotechnol. 2013, 13, 37. [CrossRef] [PubMed]

168. Lagaron, J.M.; Cabedo, L.; Cava, D.; Feijoo, J.L.; Gavara, R.; Gimenez, E. Improving packaged food quality and safety. Part 2: Nanocomposites. Food Addit. Contam. 2005, 22, 994-998. [CrossRef] [PubMed]

169. Camargo, P.H.C.; Satyanarayana, K.G.; Wypych, F. Nanocomposites: Synthesis, structure, properties and new application opportunities. Mater. Res. 2009, 12, 1-39. [CrossRef]

170. Küçükçobanoğlu, Y.; Aktaş, L.Y. Plants as a Nanocomposite Source and Field of Application. Marmara J. Pure Appl. Sci. 2018, 30, 429-436. (In Turkish) [CrossRef]

171. Draz, M.S.; Fang, B.A.; Zhang, P.; Hu, Z.; Gu, S.; Weng, K.C.; Gray, J.W.; Chen, F.F. Nanoparticle-mediated systemic delivery of siRNA for treatment of cancers and viral infections. Theranostics 2014, 4, 872. [CrossRef]

172. Mu, L.; Seow, P.H. Application of TPGS in polymeric nanoparticulate drug delivery system. Colloids Surfaces $B$ Biointerfaces 2006, 47, 90-97. [CrossRef]

173. Singh, P.; Singh, R.; Borthakur, A.; Srivastava, P.; Srivastava, N.; Dhanesh, T.D.; Mishra, P.K. Effect of nanoscale $\mathrm{TiO}_{2}$-activated carbon composite on Solanum lycopersicum (L.) and Vigna radiata (L.) seeds germination. Energ. Ecol. Environ. 2016, 1, 131-140. [CrossRef]

174. Liu, A.X.; Lu, Q.M.; Cao, Y.J.; Liao, Z.W.; Xu, Q.H. Effects of composite nanomaterials on rice growth. Plant Nutr. Fertil. Sci. 2007, 2, 344-347. (In Chinese)

175. Abdel-Aziz, H.M.M.; Hasaneen, M.N.A.; Omer, A.M. Nano chitosan-NPK fertilizer enhances the growth and productivity of wheat plants grown in sandy soil. Span. J. Agric. Res. 2016, 14, e0902. [CrossRef]

176. Yılmaz, U.; Evci, C. Future of Composite Materials in Aerospace and Defence Industry. Def. Sci. J. 2015, 14, 77-109. (In Turkish)

Publisher's Note: MDPI stays neutral with regard to jurisdictional claims in published maps and institutional affiliations.

(C) 2020 by the authors. Licensee MDPI, Basel, Switzerland. This article is an open access article distributed under the terms and conditions of the Creative Commons Attribution (CC BY) license (http://creativecommons.org/licenses/by/4.0/). 